\title{
WEIGHTED BOUNDARY LIMITS OF \\ THE GENERALIZED KOBAYASHI-ROYDEN METRICS ON WEAKLY PSEUDOCONVEX DOMAINS
}

\author{
JIYE YU
}

\begin{abstract}
The purpose of this paper is to study the existence of weighted boundary limits of the generalized Kobayashi-Royden metrics on weakly pseudoconvex domains in $\mathbb{C}^{n}$ and to explore the connections between the limits and the Levi invariants. The main result extends Graham's result on strongly pseudoconvex domains to a large class of weakly pseudoconvex domains.
\end{abstract}

\section{INTRODUCTION}

The Kobayashi/Royden (or simply Kobayashi) metric has proved to be very useful in complex analysis of several variables (cf. [BP1-2], [D-F], [J-P], [KR34]). The asymptotic boundary behavior of the Kobayashi metric has been a major area of study. In 1975, I. Graham [GR] obtained a precise weighted boundary limits (in terms of the Levi form) of the Kobayashi metric for strongly pseudoconvex domains. There have been many estimates for the metric on several classes of weakly pseudoconvex domains ever since (cf. [CA1], [CHE], [CHO], [D-F], [FR], [HE], [KR2]). In particular, the sharp bounds for the metric on pseudoconvex domains of finite type in $\mathbb{C}^{2}$ [CA1], smoothly bounded convex domains of finite type in $\mathbb{C}^{n}(n \geq 2)$ [CHE] and decoupled domains of finite type $[\mathrm{HE}]$ are obtained in terms of small/large constants. For general weakly pseudoconvex domains of finite type there are no sharp bounds known. As a matter of fact, the usual sharp lower estimates for the Kobayashi metric as in [CA1] do not hold on a general domain of finite type unless the regular type of the domain is upper semicontinuous [YU2]. On the other hand, it remains an open problem whether some analogue of Graham's results still hold on weakly pseudoconvex domains, even on domains in $\mathbb{C}^{2}$.

In this paper we intend to study the same problem for the generalized Kobayashimetrics on weakly pseudoconvex domains. Our focus here is again on the precise relationship between the (weighted) boundary limits of the metrics and the Levi invariants of the domain, in the same spirit of Graham's result in [GR]. The main difficulty in the case of weakly pseudoconvex domain is that the local Levi geometry of the domain is in general much more complicated and is still not well understood. In particular there is no universal model for

Received by the editors March 8, 1994 and, in revised form, June 6, 1994.

1991 Mathematics Subject Classification. Primary 32F15, 32H15.

Key words and phrases. Pseudoconvex, finite type, multitype, h-extendible, generalized Kobayashi metrics. 
all weakly pseudoconvex domains to compare with. To overcome this difficulty, we first deform the domain with respect to its multitype and then blow it up to a taut (but unbounded model) domain. To obtain the desired limits, we have to explore the stability and the localization problem for the metrics. The advantage of the method is that it is applicable not only to the Kobayashi-like metrics, but also to other invariant objects (cf. [K-Y]). The main result of this paper extends Graham's result in [GR] to a very large class of weakly pseudoconvex domains, called h-extendible domains, which includes almost all the interesting domains mentioned above. Roughly speaking, the h-extendible domains are the domains enjoying certain "bumping property" (precise definition is given in $\S 3$ ). As a matter of fact, the h-extendible domains are exactly those pseudoconvex domains on which Catlin's multitypes agree with D'Angelo's q-types (see [YU4] for details). It is worth mentioning that the limit in our main theorem might be the most explicit one for a general weakly pseudoconvex domain (see the final remark of the paper).

The paper is organized as follows: in $\S 1$ we recall the definitions of various types and the (higher order) Kobayashi metrics and state our main theorem. In $\S 2$, we discuss the stability property and the localization for the Kobayashi metrics. In $\S 3$ we introduce a class of models called $h$-extendible models and study the global geometry of the models. In $\S 4$, we define the h-extendibility and study the local geometry of h-extendible domains, in particular, we obtain a nice bumping theorem. In the final section, we blow up a taut domain near an h-extendible point using a scaling method and apply the localization-stability result to conclude the proof of our main theorem. Several important special cases of the main theorem are also discussed.

Acknowledgments. This work forms part of my dissertation at Washington University in St. Louis. I am very grateful to my advisor Steven Krantz for his guidance and invaluable suggestions. I would also like to thank Professors B. Stensones, J. D'Angelo, A. Noell and K. Kim for their interest and for helpful comments.

\section{Statement of MAIN Result}

Let us first recall the definitions of the relevant metrics. In what follows $\Delta$ denotes the unit disc in $\mathbb{C}, H(\Delta, \Omega)$ is the space of complex analytic discs in $\Omega$ (i.e., the set of holomorphic mappings from $\Delta$ to $\Omega$ ) and $\nu(f)$ stands for the order of vanishing of $f-f(0)$ at 0 .

Definition 1.1. Let $\Omega$ be a domain in $\mathbb{C}^{n}, z \in \Omega, X \in T_{z} \Omega \approx \mathbb{C}^{n}$. The Kobayashi metric at $(z, X)$ is defined by [KO1], [ROY]:

$$
F_{\Omega}^{K}(z, X)=\inf \left\{1 / \lambda: \lambda>0, \exists \varphi \in H(\Delta, \Omega), \varphi(0)=z, \varphi^{\prime}(0)=\lambda X\right\} .
$$

More generally, we define the higher order Kobayashi metrics as follows [YU2]: For each integer $k \geq 1$, we define

$$
\begin{aligned}
F_{\Omega}^{k}(z, X)=\inf \{1 / \lambda: \lambda>0, \exists \varphi \in H(\Delta, \Omega) \text { with } \varphi(0) & =z, \\
\nu(\varphi) & \left.\geq k, \varphi^{(k)}(0)=k ! \lambda X\right\} .
\end{aligned}
$$

Clearly $F_{\Omega}^{1}$ is just the Kobayashi metric. Throughout the paper we will use the notation $F_{\Omega}$ to denote any one of the higher order Kobayashi metrics, except 
where it is specified otherwise, and by "the Kobayashi metrics" we mean all the higher order Kobayashi metrics.

The most important property of $F_{\Omega}$ is that it decreases under holomorphic mappings, namely, if $f: \Omega_{1} \rightarrow \Omega_{2}$ is a holomorphic mapping, $z \in \Omega_{1}, X \in$ $\mathbb{C}^{n}$, then we have

$$
F_{\Omega_{2}}\left(f(z), f_{*}(X)\right) \leq F_{\Omega_{1}}(z, X) .
$$

In particular the metrics are biholomorphically invariant and if $\Omega_{1} \subset \Omega_{2} \subset \mathbb{C}^{n}$ are two domains, then $F_{\Omega_{1}}(z, X) \geq F_{\Omega_{2}}(z, X)$ for all $z \in \Omega_{1}$ and $X \in \mathbb{C}^{n}$. As shown in [YU5], the higher order Kobayashi metrics share many important properties of the standard Kobayashi metric, for instance, they are continuous on taut domains. Further, the higher order Kobayashi metrics have closer relation with the variety type than the standard Kobayashi metric [YU2]. Some further properties of the higher order Kobayashi metrics will be discussed in $\S 2$.

Next we recall the various notions of type (cf. [DA1-2], [CA2], [KON]).

Definition 1.2. Let $p$ be a smooth boundary point of the domain $\Omega$ and $\rho$ a local defining function for $\Omega$. The variety type $\Delta_{1}(p)$ of $p$ (relative to the boundary $\partial \Omega$ ) can be defined as follows:

$$
\Delta_{1}(p)=\sup \left\{\frac{\nu(\rho \circ \varphi)}{\nu(\varphi)}: \varphi \in H\left(\mathbb{C}^{n}, \Delta\right) \backslash\{0\}, \varphi(0)=p\right\} .
$$

If we require that $\varphi^{\prime}(0) \neq 0$ in the definition of $\Delta_{1}(p)$, then we obtain the regular type of $p$, denoted by $\Delta_{1}^{r}(p)$. Clearly we always have $\Delta_{1}^{r}(p) \leq \Delta_{1}(p)$, but generally they are not equal (for examples cf. [DA2], [KR1]).

A smooth domain is said to be of finite type if $\Delta_{1}(p)<\infty$ for all $p \in \partial \Omega$, the boundary of $\Omega$.

Now we recall Catlin's multitype [CA2]. Let $\Gamma_{n}$ denote the set of all $n$-tuples of numbers $\mu=\left(\mu_{1}, \mu_{2}, \ldots, \mu_{n}\right)$ with $1 \leq \mu_{i} \leq \infty$ such that

(i) $\mu_{1} \leq \mu_{2} \leq \cdots \leq \mu_{n}$,

(ii) for each $k$, either $\mu_{k}=\infty$ or there is a set of nonnegative integers $a_{1}, \ldots, a_{k}$, with $a_{k}>0$ such that $\sum_{j=1}^{k} a_{j} / \mu_{j}=1$.

An element of $\Gamma_{n}$ will be referred to as a word. The set of words can be ordered lexicographically, i.e. if $\mu^{\prime}=\left(\mu_{1}^{\prime}, \ldots, \mu_{n}^{\prime}\right)$ and $\mu^{\prime \prime}=\left(\mu_{1}^{\prime \prime}, \ldots, \mu_{n}^{\prime \prime}\right)$, then $\mu^{\prime}<\mu^{\prime \prime}$ if for some $k, \mu_{j}^{\prime}=\mu_{j}^{\prime \prime}$ for all $j<k$, but $\mu_{k}^{\prime}<\mu_{k}^{\prime \prime}$.

A word $\mu \in \Gamma_{n}$ is said to be distinguished if there exist holomorphic coordinates $\left(z_{1}, z_{2}, \ldots, z_{n}\right)$ about $p$ with $p$ mapped to the origin such that

$$
\text { If } \sum_{i=1}^{n} \frac{\alpha_{i}+\beta_{i}}{\mu_{i}}<1, \quad \text { then } D^{\alpha} \bar{D}^{\beta} r(p)=0 .
$$

Here $D^{\alpha}$ and $\bar{D}^{\beta}$ denote the partial differential operators

$$
\frac{\partial^{|\alpha|}}{\partial z_{1}^{\alpha_{1}} \cdots \partial z_{n}^{\alpha_{n}}} \quad \text { and } \frac{\partial^{|\beta|}}{\partial \bar{z}_{1}^{\beta_{1}} \cdots \partial \bar{z}_{n}^{\beta_{n}}} \quad \text { respectively. }
$$

Definition 1.3. The multitype $\mathscr{M}(p)$ is defined to be the least word $\mathscr{M}=$ $\left(\mu_{1}, \ldots, \mu_{n}\right)$ in $\Gamma_{n}$ (in the lexicographic sense) such that $\mathscr{M} \geq \mu$ for every distinguished word $\mu$. 
Since $p$ is a smooth point, it is easy to see that the first entry $\mu_{1}$ in $\mathscr{M}(p)$ is always 1 .

It is proved in [CA2] that if $p$ is of finite type, then every component of the multitype of $p$ is finite. Further, if $p$ is of finite type, then there are local holomorphic coordinates about $p$ such that $p=0$ and (1.1) holds; such holomorphic coordinates will be referred to as distinguished coordinates.

The main result of this paper is:

Main Theorem. Let $\Omega$ be a taut domain in $\mathbb{C}^{n+1}$ and let $p \in \partial \Omega$ be of finite type with multitype $\mathscr{M}(p)=\left(1, m_{1}, \ldots, m_{n}\right)$. Suppose that $(\Omega, p)$ is an $h$ extendible pointed domain. Then for any nontangential cone $\Gamma$ in $\Omega$ with vertex at $p$ and any $\mathbb{C}^{n+1}$-valued function $V(z)$ which is continuous at $p$, we have

$$
\lim _{\Omega \cap \Gamma \ni z \rightarrow p} F_{\Omega}\left(z,\left(H_{p}\right)_{* z}^{-1}\left(\Pi_{d}\right)_{* q}\left(H_{p}\right)_{* z} V(z)\right)=F_{D_{p}}\left(0,\left(H_{p}\right)_{* p} V(p)\right) .
$$

Here $H_{p}$ is distinguished coordinates as defined above, $D_{p}=\{(z, w)$ : $\operatorname{Re} w+P(z)<1\}$ is the model associated with the pointed domain $(\Omega, p)$ in the distinguished coordinates $q=H_{p}(z)$ and $\Pi_{d *}=\operatorname{diag}\left[d^{1 / m_{n}}, \ldots, d^{1 / m_{1}}, d\right]$ with $d=\operatorname{dist}\left(q, \partial\left(H_{p}(\Omega)\right)\right)$. Roughly speaking, the equation $(*)$ says that the weighted boundary limit of any higher order Kobayashi metric on an hextendible domain is exactly the value of the metric of the associated model at the interior point 0 . We will explain the notions of models and extendible domains in $\S 3$ and $\S 4$.

One special feature of the main theorem is that it provides a formula to find the precise relationship between the Levi invariants of the domain and the weighted boundary limits of the Kobayashi metrics. For instance, if the principal part $P(z)$ of the defining function is circular, then we can actually calculate through $(*)$ the precise boundary asymptotic limits for the metrics (see Corollary 5.4). This yields in particular the exact asymptotic boundary behavior of the Kobayashi metric for egg domains [BKM] as well as for strongly pseudoconvex domains [GR].

It is shown in $\S 3$ that the models associated with an h-extendible domain are hyperbolic, thus the right-hand side of $(*)$ is always positive. Accordingly, we can deduce from $(*)$ sharp lower and upper bounds for the Kobayashi metrics on any h-extendible domain. Since the pseudoconvex domains of finite type in $\mathbb{C}^{2}$ and convex domains of finite type in $\mathbb{C}^{n}$ are special examples of h-extendible domains, we then obtain sharp bounds of the metrics on these domains. In this sense the main theorem unifies many existing results. Finally we remark that the finite type condition in the main theorem is necessary (cf. [YU2]).

\section{Stability AND LOCALIZATION OF THE Kobayashi METRICS}

Two of the crucial elements in our approach to the asymptotic boundary behavior problem are the stability and localization of the invariant metrics. Let $\left\{D_{j}\right\}_{j \geq 1}, D$ be a family of domains in $\mathbb{C}^{n}$ such that $D_{j} \rightarrow D$ as $j \rightarrow \infty$ in the sense that

$$
\lim _{j \rightarrow \infty} d\left(\partial D_{j}, \partial D\right)=0 .
$$

Here $d(A, B)$ stands for the Hausdorff distance between the two sets $A, B$. Let $F_{D_{j}}, F_{D}$ denote the Kobayashi metrics for $D_{j}, D$ respectively. Then we are interested in the following stability problem: 


$$
\lim _{j \rightarrow \infty} F_{D_{j}}(z, X)=F_{D}(z, X) \quad \forall(z, X) \in D \times \mathbb{C}^{n} ?
$$

This stability problem has been studied from several points of view by many people (cf. [KA], [WR], [GK1]). All the known results are valid only for bounded domains (or compact complex manifolds). Since we will deal with unbounded model domains, we need to establish a stability property for all higher order Kobayashi metrics allowing perturbations of unbounded domains.

Theorem 2.1. Let $D_{j}, D$ be a family of domains in $\mathbb{C}^{n}$ satisfying the condition (2.1). Let $D$ be a taut domain. If, there exists another taut domain $\Omega$ such that $D_{j} \subset \Omega$ for all large $j$, then we have

$$
\lim _{j \rightarrow \infty} F_{D_{j}}^{k}(z, X)=F_{D}^{k}(z, X) \quad \forall(z, X) \in D \times \mathbb{C}^{n}, \quad k \geq 1 .
$$

Moreover, the convergence takes place uniformly over compact subsets of $D \times \mathbb{C}^{n}$.

Here $F_{D}^{k}$ denotes the $k$ th order Kobayashi metric and $k \geq 1$ is arbitrary. Proof. Fix $k \geq 1, E \subset \subset D$ and $G \subset \subset \mathbb{C}^{n}$. Assume to the contrary that $F_{D_{j}}^{k}(z, X)$ does not converge to $F_{D}^{k}(z, X)$ uniformly on $E \times G$. Then there exist $\varepsilon_{0}>0$, a sequence of integers $\left\{j_{\ell}\right\}_{\ell \geq 1}$, a sequence of points $\left\{z_{j_{\ell}}\right\} \subset$ $E \subset \subset D_{j_{\ell}}$ and a sequence $\left\{X_{j_{\ell}}\right\} \subset \subset G$ such that

$$
\left|F_{D_{j_{\ell}}}^{k}\left(z_{j_{\ell}}, X_{j_{\ell}}\right)-F_{D}^{k}\left(z_{j_{\ell}}, X_{j_{\ell}}\right)\right|>\varepsilon_{0} .
$$

By the homogeneity of the Kobayashi metrics $F^{k}(z, X)$ in the second variable, it may be assumed that $\left|X_{j_{\ell}}\right|=1$ for all $\ell \geq 1$. Further, it may also be assumed that $z_{j_{\ell}} \rightarrow z_{0} \in \bar{E}, X_{j_{\ell}} \rightarrow X_{0} \in \bar{G}$ as $j \rightarrow \infty$. Since $D$ is taut, $F_{D}^{k}(z, X)$ is jointly continuous in $(z, X)$ (cf. [YU5]). Therefore we have

$$
\left|F_{D_{j_{\ell}}}^{k}\left(z_{j_{\ell}}, X_{j_{\ell}}\right)-F_{D}^{k}\left(z_{0}, X_{0}\right)\right|>\varepsilon_{0} / 2 \quad \text { for } \ell \text { sufficiently large. }
$$

By Definition 1.1 , for any $\eta \in(0,1)$, there exists $\varphi_{j_{\ell}} \in H\left(\Delta, D_{j_{\ell}}\right)$ such that $\varphi_{j_{\ell}}(0)=z_{j_{\ell}}, \nu\left(\varphi_{j_{\ell}}\right)=k, \varphi_{j_{\ell}}^{(k)}(0)=k ! \lambda_{j_{\ell}} X_{j_{\ell}}$, where $\lambda_{j_{\ell}}>0$, and

$$
F_{D_{j_{\ell}}}^{k}\left(z_{j_{\ell}}, X_{j_{\ell}}\right) \geq \frac{1}{\lambda_{j_{\ell}}}-\eta
$$

Claim. Every subsequence of the $\left\{\varphi_{j_{\ell}}\right\}$ has itself a subsequence converging to some element $\psi \in H(\Delta, D)$ such that $\psi(0)=z_{0}, \nu(\psi)=k$ and $\psi^{(k)}(0)=$ $k ! \lambda X_{0}$ for some $\lambda>0$. As a result of the claim, we will obtain

$$
\varliminf_{\ell \rightarrow \infty} F_{D_{j_{\ell}}}^{k}\left(z_{j_{\ell}}, X_{j_{\ell}}\right) \geq F_{D}^{k}\left(z_{0}, X_{0}\right)-\eta .
$$

First we check the claim. By hypothesis, $D_{j} \subset \Omega$ for all $j \geq 1$, we have $\varphi_{j_{\ell}} \in H(\Delta, \Omega)$ for all $\ell$. By the tautness of $\Omega$, every subsequence of $\left\{\varphi_{j_{\ell}}\right\}$ has itself a subsequence either converging to some element $\psi \in H(\Delta, \Omega)$ or compactly divergent. Since $\varphi_{j_{\ell}}(0)=z_{j_{\ell}} \rightarrow z_{0} \in D$ as $\ell \rightarrow \infty$, only the first possibility could happen. Moreover, because $\varphi_{j_{\ell}}(\Delta) \subset D_{j_{\ell}} \rightarrow D$, it follows $\psi(\Delta) \subset \bar{D}$. But $D$ is taut and $\psi(0)=z_{0} \in D$, so we must have $\psi(\Delta) \subset D$. This verifies the claim. It follows from the claim and Definition 1.1 that

$$
F_{D}^{k}\left(z_{0}, X_{0}\right) \leq \frac{k !}{\left|\psi^{(k)}(0)\right|} \quad \text { for any such } \psi .
$$

This implies (2.6). 
Now we seek a contradiction. By the tautness of $D$, there exists an extremal disc $\varphi \in H(\Delta, D)$ for $F_{D}^{k}\left(z_{0}, X_{0}\right)$. Namely, $\varphi(0)=z_{0}, \nu(\varphi)=k$ and $\varphi^{(k)}(0)=k ! \lambda X_{0}$ with $\lambda=1 / F_{D}^{k}\left(z_{0}, X_{0}\right)$. Note that $\lambda \in(0, \infty)$. For $\eta \in(0,1)$ fixed, set, for $\zeta \in \Delta$,

$$
\psi_{j_{\ell}}^{\eta}(\zeta)=: \varphi((1-\eta) \zeta)+\lambda(1-\eta)^{k} \zeta^{k}\left(X_{j_{\ell}}-X_{0}\right)+\left(z_{j_{\ell}}-z_{0}\right) .
$$

Clearly $\psi_{j,}^{\eta} \in H\left(\Delta, \mathbb{C}^{n}\right)$. Since $\varphi(\Delta) \subset D$ and $(1-\eta) \bar{\Delta} \subset \subset \Delta$, we see that $\varphi((1-\eta) \bar{\Delta})$ is compact in $D$. It follows then from $z_{j_{\ell}} \rightarrow z_{0}, X_{j_{\ell}} \rightarrow z_{0}$ and $D_{j_{\ell}} \rightarrow D$ that $\psi_{j_{\ell}}^{\eta}(\Delta) \subset D_{j_{\ell}}$ for all sufficiently large $\ell$. That is, $\psi_{j_{\ell}}^{\eta} \in$ $H\left(\Delta, D_{j_{\ell}}\right)$. Furthermore, by construction, we have $\psi_{j_{\ell}}^{\eta}(0)=z_{j_{\ell}}, \nu\left(\psi_{j_{\ell}}^{\eta}\right)=k$ and $\left(\psi_{j_{\ell}}^{\eta}\right)^{(k)}(0)=k !(1-\eta)^{k} \lambda X_{j_{\ell}}$. It follows from the definition that

$$
F_{D_{j_{\ell}}}^{k}\left(z_{j_{\ell}}, X_{j_{\ell}}\right) \leq \frac{1}{(1-\eta)^{k} \lambda}=\frac{1}{(1-\eta)^{k}} F_{D}^{k}\left(z_{0}, X_{0}\right) \quad \text { for all large } \ell .
$$

Letting $\eta \rightarrow 0^{+}$after taking limsup with respect to $\ell$ in (2.7), we finally obtain

$$
\varlimsup_{\ell \rightarrow \infty} F_{D_{j_{\ell}}}^{k}\left(z_{j_{\ell}}, X_{j_{\ell}}\right) \leq F_{D}^{k}\left(z_{0}, X_{0}\right) .
$$

Obviously (2.8) together with (2.6) contradicts (2.4). This finishes the proof.

The stability problem for the Bergman kernel and metric is studied in [GK2], [RAM], [YU3].

Next we discuss the localization of the Kobayashi metric and the higher order Kobayashi metrics.

Theorem 2.2. Let $D$ be a taut domain in $\mathbb{C}^{n}$ and $p \in \partial D$ a fixed boundary point. Assume that the boundary of $D$ does not contain any nontrivial analytic variety through $p$. Let $U$ be a neighborhood of $p$ in $\mathbb{C}^{n}$ such that $D_{1}=D \cap U$ and $X(z): D_{1} \rightarrow \mathbb{C}^{n} \backslash\{0\}$ a continuous mapping. Then we have

$$
\lim _{D_{1} \ni z \rightarrow p} \frac{F_{D_{1}}(z, X(z))}{F_{D}(z, X(z))}=1 .
$$

Moreover, the convergence is uniform if the size of $U$ is fixed.

This theorem is a generalization of a localization result in [F-R]. It can be proved by using the normal family arguments (cf. [YU5]).

Remark. The localization of the Kobayashi metric for a smooth strongly pseudoconvex domain was first studied by Graham [GR].

An immediate consequence of Theorem 2.2 is the following

Corollary 2.3. Suppose that $D$ is a taut domain in $\mathbb{C}^{n}$ and $p \in \partial D$ is of finite type. Let $D_{1}, X(z)$ be given as in Theorem 2.2. Then the localization (2.9) holds.

\section{TAUTNESS OF UNBOUNDED MODELS}

The local geometry of a strongly pseudoconvex domain is often not much different from that of the unit ball [FE]. Thus the unit ball can be viewed as 
a universal model for all strongly pseudoconvex domains. For weakly pseudoconvex domains, however, it is generally impossible to find even a reasonable family of universal models. In this section, we will concentrate on a class of models, namely the h-extendible models, which will represent a fairly general class of weakly pseudoconvex domains.

We call a multi-index $\Lambda=\left(\lambda_{1}, \ldots, \lambda_{n}\right)$ a multiweight if $0<\lambda_{1} \leq \lambda_{2} \cdots \leq$ $\lambda_{n}<1$. The set of all such multiweights in $\mathbb{R}^{n}$ is denoted by $\mathscr{W}_{n}$.

Definition 3.1 Let $f(z)$ be a function on $\mathbb{C}^{n}$ and $\Lambda=\left(\lambda_{1}, \lambda_{2}, \ldots, \lambda_{n}\right) \in \mathscr{W}_{n}$ a multiweight. For any real number $t \geq 0$, set

$$
\pi_{t}(z)=\left(t^{\lambda_{1}} z_{1}, t^{\lambda_{2}} z_{2}, \ldots, t^{\lambda_{n}} z_{n}\right) \quad \forall z \in \mathbb{C}^{n} .
$$

We say that $f$ is $\Lambda$-homogeneous with weight $\alpha$ if $f\left(\pi_{t}(z)\right)=t^{\alpha} f(z)$ for every $t \geq 0$ and $z \in \mathbb{C}^{n}$. In case $\alpha=1$, then $f$ is simply called $\Lambda$-homogeneous.

One such example that we will refer to frequently in what follows is:

$$
\sigma(z)=\sigma_{\Lambda}(z)=: \sum_{i=1}^{n}\left|z_{i}\right|^{1 / \lambda_{i}}, \quad \text { for } \Lambda=\left(\lambda_{1}, \ldots, \lambda_{n}\right) .
$$

Now let $\Lambda$ be a multiweight and $P$ a real-valued $\Lambda$-homogeneous function on $\mathbb{C}^{n}$. Set

$$
D_{\Lambda} \equiv D_{\Lambda, P}=:\left\{(z, w) \in \mathbb{C}^{n} \times \mathbb{C}: \operatorname{Re} w+P(z)<0\right\} .
$$

We will call $D_{\Lambda}$ a model (domain) if $P$ is plurisubharmonic (p.s.h.) but not pluriharmonic (p.h.). In case $P$ is a polynomial, we will call it a polynomial model. We will consider primarily polynomial models in this section, even though most of the results are also valid on non-polynomial models.

Definition 3.2. A model $D_{\Lambda}=\{(z, w): \operatorname{Re} w+P(z)<0\}$ (hereinafter, whenever we write $(z, w)$ we mean $z \in \mathbb{C}^{n}$ and $\left.w \in \mathbb{C}\right)$ is said to be positive if $P(z)>0$ whenever $z \neq 0$. It is called a homogeneous model if $P(z)$ is homogeneous in the usual sense, namely, $\Lambda=(1 / m, \ldots, 1 / m)$ with $m$ integer. The model $D_{\Lambda}$ is called decoupled if $P$ can be written as $P(z)=\sum_{k=1}^{n} P_{k}\left(z_{k}\right)$, where each $P_{k}\left(z_{k}\right)$ is a real homogeneous subharmonic polynomial containing no pure harmonic monomials. All these models are examples of the following class of models.

Definition 3.3. Let $D_{\Lambda}=\{(z, w): \operatorname{Re} w+P(z)<0\}$ be a model. Then $D_{\Lambda}$ is called $h$-extendible if there exists a $\Lambda$-homogeneous $C^{1}$ function $a(z)$ on $\mathbb{C}^{n} \backslash\{0\}$ satisfying the following conditions:

(i) $a(z)>0$ whenever $z \neq 0$;

(ii) $P(z)-a(z)$ is p.s.h. on $\mathbb{C}^{n}$.

We will call $a(z)$ a bumping function.

Remark. (1) In [YU4], several equivalent conditions to the h-extendibility will be given. (2) Note that the condition (ii) is equivalent to the following condition:

(ii)' For any $\varepsilon \in[0,1], P(z)-\varepsilon a(z)$ is also p.s.h. on $\mathbb{C}^{n}$. This is because $P-\varepsilon a=(1-\varepsilon) P+\varepsilon(P-a)$. (3) It is easy to derive from (i) and $\Lambda$-homogeneity that there is a constant $C>0$ such that

$$
C^{-1} \sigma_{\Lambda}(z) \geq a(z) \geq C \sigma_{\Lambda}(z), \quad \text { for all } z \in \mathbb{C}^{n} .
$$


Here $\sigma_{\Lambda}$ is given by (3.2). (4) The idea of extendibility made its first appearance, in different form, in [D-F]. Related concepts are also introduced in [D-L] [DHO] and [HE].

Let us look at a few examples.

Example 3.4. Every positive model is h-extendible. The converse is not true, as the next example shows.

Example 3.5. Every model in $\mathbb{C}^{2}$ is h-extendible. For example, consider $D=$ $\left\{(z, w): \operatorname{Re} w+|z|^{4}-4 / 3 \operatorname{Re}\left(z \bar{z}^{3}\right)<0\right\}$. Notice that this model is not positive. Moreover, there is no smooth bumping function.

Example 3.6. Every decoupled model is h-extendible.

A generalization of Example 3.5 is the following fact proved by Noell [NO]:

Proposition 3.7. Let $D_{\Lambda}=\{(z, w): \operatorname{Re} w+P(z)<0\}$ be a homogeneous polynomial model in $\mathbb{C}^{n+1}$ with $\Lambda=(1 / m, \ldots, 1 / m)$. If $P(z)$ is not harmonic along any complex line through 0 , then $D_{\Lambda}$ is h-extendible.

Of course not every model is h-extendible. For example $\left\{\left(z_{1}, z_{2}, z_{3}\right)\right.$ : $\left.\operatorname{Re} z_{3}+\left|z_{1}^{2}-z_{2}^{3}\right|^{2}<0\right\}$ and $\left\{\left(z_{1}, z_{2}, z_{3}\right): \operatorname{Re} z_{3}+\left|z_{1} z_{2}\right|^{2}<0\right\}$. Note that, in these two models, there are nontrivial complex analytic varieties through 0 that are contained in the boundaries. This situation cannot happen to the $\mathrm{h}$ extendible models. In fact, it can be shown that any h-extendible polynomial model is of finite type [YU5].

Now we consider the tautness of models. Since our models are not biholomorphic to bounded domains in general, we cannot apply the known criterion (cf. [K-R], [DE]). On the other hand, it is easy to see that the model $\left\{\left(z_{1}, z_{2}, w\right): \operatorname{Re} w+\left|z_{1}^{2}-z_{2}^{3}\right|^{2}<0\right\}$ is not taut. However, we will show that all h-extendible models are indeed taut.

Let us first consider the hyperbolicity of h-extendible models.

Lemma 3.8. (Hörmander) Let $\Omega$ be a pseudoconvex open set in $\mathbb{C}^{n}$ and let $\varphi$ be any plurisubharmonic function on $\Omega$. For every $(0,1)$ form $g \in L^{2}(\Omega, \varphi)$ with $\bar{\partial} g=0$ there is a solution $u \in L^{2}(\Omega$, loc) of the equation $\bar{\partial} u=g$ such that

$$
\int_{\Omega}|u|^{2} e^{-\varphi}\left(1+|z|^{2}\right)^{-2} d V \leq \int_{\Omega}|g|^{2} e^{-\varphi} d V
$$

Here $d V$ denotes the Euclidean volume form on $\Omega$.

Let $\Lambda=\left(\lambda_{1}, \ldots, \lambda_{n}\right)$ be a multiweight and let $\alpha>0$ be fixed. The following fact about weighted homogeneous function can be proved easily.

Lemma 3.9. For any $C^{1}$ smooth $\Lambda$-homogeneous function $f(z)$ on $\mathbb{C}^{n} \backslash\{0\}$ with weight $\beta \in[0, \alpha]$, there exist constants $C>0$ and $k=\max _{i}\left\{\alpha-\lambda_{i}\right\}$ such that

$$
\left|f\left(z+z^{\prime}\right)-f(z)\right| \leq C \quad \text { if }\left|z^{\prime}\right| \leq \frac{1}{(1+\sigma(z))^{k}} .
$$

Now let $D_{\Lambda}=\{(z, w): \operatorname{Re} w+P(z)<0\}$ be an h-extendible model in $\mathbb{C}^{n+1}$ with a bumping function $2 a$. Put

$$
\varphi(z)=P(z), \quad \psi(z)=P(z)-a(z), \quad k=\max _{i}\left\{1-\lambda_{i}\right\} .
$$

Then, combining Lemma 3.9 and (3.3), we obtain 
Proposition 3.10. Let $\varphi, \psi$ be given as above. Then

(1) There exists a constant $C>0$ such that

$$
\left|\psi\left(z+z^{\prime}\right)-\psi(z)\right| \leq C \quad \text { if } \quad\left|z^{\prime}\right| \leq \frac{1}{(1+\sigma(z))^{k}} .
$$

(2) There exists constant a $c>0$ such that

$$
\varphi(z) \geq \psi(z)+2 c \sigma(z), \quad \text { if }|z| \text { is sufficiently large. }
$$

Now we can give a proof of the Carathéodory hyperbolicity. By definition it is equivalent to the following:

Theorem 3.11. Suppose that $D=: D_{\Lambda}=\{(z, w): \operatorname{Re} w+P(z)<0\}$ is an h-extendible model. Then $A^{0}(D)$ separates points of $D$. That is, $D$ is Carathéodory hyperbolic.

Here $A^{0}(D)$ is the algebra of holomorphic functions on $D$ which are also continuous on $\bar{D}$.

Proof. Let $\left(z_{i}, w_{i}\right) \in \mathbb{C}^{n} \times \mathbb{C}, i=1,2$, be any two different points in $D$. There are two cases to consider: $z_{1} \neq z_{2}$ and $z_{1}=z_{2}$. (a) If $z_{1} \neq z_{2}$, then set $\delta=\left|z_{1}-z_{2}\right|$. Choose a real function $\theta \in C^{\infty}\left(\mathbb{C}^{n}\right)$ such that $\theta(z)=0$ if $|z| \leq \frac{1}{2}$ and $\theta(z)=1$ if $|z| \geq \frac{3}{4}$. Let $\chi(z)=1-\theta\left(\left(z-z_{2}\right) / \delta\right)$ and $g(z)=\bar{\partial} \chi(z)$. Define a p.s.h. function

$$
\varphi_{1}(z)=\psi(z)+2 n\left(\ln \left|z-z_{1}\right|+\ln \left|z-z_{2}\right|\right) .
$$

Here $\psi=P-a$ as in Proposition 3.10. Applying Lemma 3.8 with the weight function $\varphi_{1}$, one gets a function $u$ such that $\bar{\partial} u=g$ and

$$
\int_{\mathbb{C}^{n}}|u|^{2} \frac{e^{-\psi(z)}}{\left(1+|z|^{2}\right)^{2}\left|z-z_{1}\right|^{2 n}\left|z-z_{2}\right|^{2 n}} d z \leq 2 \int_{\mathbb{C}^{n}}|g|^{2} \frac{e^{-\psi(z)}}{\left|z-z_{1}\right|^{2 n}\left|z-z_{2}\right|^{2 n}} d z .
$$

It follows from the convergence of the integral that $u\left(z_{1}\right)=u\left(z_{2}\right)=0$. Set $f(z)=\chi(z)-u(z)$. Then $f$ is an entire function with $f\left(z_{1}\right)=1$ and $f\left(z_{2}\right)=$ 0 . For $\left|z-z_{1}\right| \geq 3 \delta$, let $B(z, r)$ denote the ball of center $z$ and radius $r=1 /(1+\sigma(z))^{k}$, where $k$ is given in Proposition 3.10. Since $u$ is holomorphic for $\left|z-z_{1}\right|>\delta$, the function $|u|^{2}$ satisfies the submean value property:

$$
|f(z)|^{2}=|u(z)|^{2} \leq \frac{1}{\operatorname{vol}(B(z, r))} \int_{B(z, r)}|u(\zeta)|^{2} d \zeta .
$$

Clearly, for all $\zeta \in B(z, r)$ we have $e^{-\psi(\zeta)} \sup _{\left|z^{\prime}\right|<r} e^{\psi\left(z+z^{\prime}\right)} \geq 1$ and

$\left(1+|\zeta|^{2}\right)^{2}\left|\zeta-z_{1}\right|^{2 n}\left|\zeta-z_{2}\right|^{2 n} \lesssim|\zeta|^{4 n+4} \lesssim|z|^{4 n+4} \lesssim(1+\sigma(z))^{m}$ for some $m>0$.

Here and in the following the symbol $A \lesssim B$ means that there is an absolute constant $C>0$ such that $A \leq C B$. Combining (3.6) and (3.7) we get

$$
\begin{aligned}
|f(z)|^{2} & =|u(z)|^{2} \\
& \lesssim \frac{1}{r^{2 n}} \sup _{\left|z^{\prime}\right|<r} e^{\psi\left(z+z^{\prime}\right)}(1+\sigma(z))^{m} \int_{B(z, r)} \frac{e^{-\psi(\zeta)}|u(\zeta)|^{2}}{\left(1+|\zeta|^{2}\right)^{2}\left|\zeta-z_{1}\right|^{2 n}\left|\zeta-z_{2}\right|^{2 n}} d V \\
& \lesssim(1+\sigma(z))^{2 n k+m} e^{\psi(z) \quad \text { by }(3.5) \text { and (1) in Proposition 3.10 }} \\
& \lesssim(1+\sigma(z))^{2 n k+m} e^{-2 c \sigma(z)} e^{P(z)} \quad \text { by (2) in Proposition 3.10 } \\
& \lesssim e^{P(z)-c \sigma(z)} \quad \text { if }|z| \text { is sufficiently large. }
\end{aligned}
$$


Thus there exists a constant $C>0$ such that for all $z \in \mathbb{C}^{n}$, we have

$$
|f(z)|^{2} \leq C e^{P(z)-c \sigma(z)} \leq C e^{P(z)}, \quad \text { since } \sigma(z) \geq 0 \forall z \in \mathbb{C}^{n} .
$$

Let $g(z, w)=e^{w} f(z)^{2}$. Then $g$ is bounded on $D$ and separates $\left(z_{1}, w_{1}\right)$, $\left(z_{2}, w_{2}\right)$.

(b) If $z_{1}=z_{2}$, then $w_{1} \neq w_{2}$. Using the same idea as above we can find an entire function $f$ so that $f\left(z_{1}\right)=1$ and $|f(z)|^{2} \lesssim e^{P(z)}$. Then we use the function $e^{w} f(z)^{2}$ to separate the two points $\left(z_{1}, w_{1}\right),\left(z_{1}, w_{2}\right)$.

Similar ideas as above can be applied to construct peaking functions for $\mathrm{h}$ extendible models (for details see [YU5]).

Since the Kobayashi metrics are larger than the Carathéodory metric [YU5], we obtain

Corollary 3.12. Any h-extendible model is hyperbolic with respect to all the higher order Kobayashi metrics.

Finally we prove the main goal of this section, namely

Theorem 3.13. Every h-extendible model is taut.

Since the defining function of an h-extendible model is p.s.h., to prove Theorem 3.13 it suffices to prove the following

Lemma 3.14. Let $D=: D_{\Lambda}$ be an h-extendible model and $\left\{f_{n}\right\}_{n \geq 1} \subset H(\Delta, D)$. Then $\left\{f_{n}\right\}_{n \geq 1}$ is a normal family in $H\left(\Delta, \mathbb{C}^{n+1}\right)$.

Proof. Let $u(z, w)=\operatorname{Re} w+P(z)-a(z)$ and $D_{1}=\{u(z, w)<0\}$, where $2 a(z)$ is a bumping function for $D$. Then $D_{1}$ is also an h-extendible model and thus is Kobayashi hyperbolic by Corollary 3.12. Let $d_{K}$ denote the Kobayashi distance on $D_{1}$. Then, by Royden [ROY], the topology induced by $d_{K}$ coincides with the one induced by the Euclidean distance. This implies in particular that every family of holomorphic mappings in $H\left(\Delta, D_{1}\right)$ is equicontinuous. Now consider $u_{n}(\zeta)=: u\left(f_{n}(\zeta)\right): \Delta \rightarrow \mathbb{R}^{-}$. There are two possibilities:

Case (1): For every $\zeta \in \Delta, \varlimsup_{n} u_{n}(\zeta)=-\infty$. Since $u_{n}(\zeta) \leq 0$, we have $\lim _{n \rightarrow \infty} u_{n}(\zeta)=-\infty, \forall \zeta \in \Delta$. In this case we will show that $\left\{f_{n}\right\}$ is compactly divergent in $\mathbb{C}^{n+1}$. To this end, take any $K \subset \subset \Delta, L \subset \subset \mathbb{C}^{n+1}$. We claim that $f_{n}(K) \cap L=\varnothing$ for $n$ large. Suppose not. Then there exists a sequence of points $\left\{\zeta_{n_{k}}\right\}$ of $K$ such that $f_{n_{k}}\left(\zeta_{n_{k}}\right) \in L$ for all $k \geq 1$. Since $K, L$ are compact, without loss of generality, we may assume that $\zeta_{n_{k}} \rightarrow \zeta_{0} \in K$ and $\left|f_{n_{k}}\left(\zeta_{n_{k}}\right)\right| \leq c$ for some constant $c>0$. Since $\left\{f_{n_{k}}\right\}$ is equicontinuous on $\Delta$, for $\varepsilon=1$ and $k$ large, we will have $\left|f_{n_{k}}\left(\zeta_{n_{k}}\right)-f_{n_{k}}\left(\zeta_{0}\right)\right| \leq 1$. It follows that

$$
\left|f_{n_{k}}\left(\zeta_{0}\right)\right| \leq\left|f_{n_{k}}\left(\zeta_{n_{k}}\right)\right|+1 \leq c+1<\infty, \quad \text { for all large } k .
$$

This contradicts our assumption that $\lim _{k \rightarrow \infty} u\left(f_{n_{k}}\left(\zeta_{0}\right)\right)=-\infty$ (since $u$ is continuous).

Case (2): There exists a $\zeta_{0} \in \Delta$ such that $\overline{\lim }_{n} u_{n}\left(\zeta_{0}\right)>-\infty$. Then there is a subsequence, without loss of generality we still denote it by $u_{n}$, such that

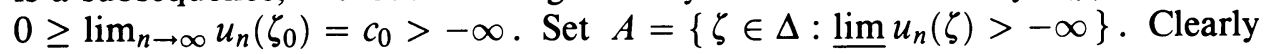
$\zeta_{0} \in A$, so $A \neq \varnothing$. 
Next we show that $A$ is an open set. As a matter of fact, if $\zeta_{0} \in A$, then there exists a constant $c \leq 0$ such that $\underline{\lim } u_{n}\left(\zeta_{0}\right)=c>-\infty$. Thus, for $\varepsilon=(1-c) / 2>0$, we can find an $n_{0} \geq 1$ such that

$$
u_{n}\left(\zeta_{0}\right) \geq c-\varepsilon=\frac{3 c-1}{2}=:-c_{1}, \quad \forall n \geq n_{0} .
$$

Note that $c_{1}>0$. Write out the last inequality using $f_{n}=\left(f_{n}^{1}, f_{n}^{2}\right)$, where $f_{n}^{1} \in \mathbb{C}^{n}, f_{n}^{2} \in \mathbb{C}$. We have

$$
\operatorname{Re} f_{n}^{2}\left(\zeta_{0}\right)+P\left(f_{n}^{1}\left(\zeta_{0}\right)\right)-a\left(f_{n}^{1}\left(\zeta_{0}\right)\right) \geq-c_{1}, \quad \forall n \geq 1 .
$$

This implies that $-a\left(f_{n}^{1}\left(\zeta_{0}\right)\right) \geq-c_{1}$, since $f_{n} \in H(\Delta, D)$. Thus by $(*)$ we see that $\left\{f_{n}^{1}\left(\zeta_{0}\right)\right\}$ is bounded, i.e., there is a constant $\lambda>0$ such that $\left|f_{n}^{1}\left(\zeta_{0}\right)\right| \leq \lambda$, for all $n \geq 1$. In light of the equicontinuity of $f_{n}$, for $\varepsilon=\lambda$, there exists a $\delta_{1}>0$ such that whenever $\left|\zeta-\zeta_{0}\right| \leq \delta_{1}$ we have $\left|f_{n}^{1}(\zeta)-f_{n}^{1}\left(\zeta_{0}\right)\right| \leq \lambda, \quad \forall n \geq 1$. It follows that

$$
f_{n}^{1}\left(\bar{\Delta}\left(\zeta_{0}, \delta_{1}\right)\right) \subset \bar{B}(0,2 \lambda)=:\left\{z \in \mathbb{C}^{n}:|z| \leq 2 \lambda\right\} .
$$

On the other hand, observe that the function $u(z, w)$ is uniformly continuous on sets of the form $K \times \mathbb{C}$, where $K \subset \subset \mathbb{C}^{n}$. Thus the equicontinuity together with (3.11) implies that $u_{n}$ is equicontinuous on $\bar{\Delta}\left(\zeta_{0}, \delta_{1}\right)$. Therefore, for $\varepsilon=c_{1}$, there is a $0<\delta \leq \delta_{1}$ such that whenever $\left|\zeta-\zeta_{0}\right| \leq \delta$, we have $\left|u_{n}(\zeta)-u_{n}\left(\zeta_{0}\right)\right| \leq \varepsilon=c_{1} \forall n \geq 1$. This implies that

$$
u_{n}(\zeta) \geq u_{n}\left(\zeta_{0}\right)-c_{1} \geq-2 c_{1}, \quad \forall n \geq 1 .
$$

Hence $\underline{\lim }_{n \rightarrow \infty} u_{n}(\zeta) \geq-2 c_{1}>-\infty$, for all $\zeta \in \Delta\left(\zeta_{0}, \delta\right)$. That is, $\Delta\left(\zeta_{0}, \delta\right) \subset$ $A$. Thus $A$ is open in $\Delta$.

Moreover, the set $\mathrm{A}$ is also closed in $\Delta$. To this end, let $\zeta_{j} \in A, j \geq 1$, and assume that $\zeta_{j} \rightarrow \zeta_{0} \in \Delta$, as $j \rightarrow \infty$. We will show that $\zeta_{0} \in A$. Assume the contrary. Then we would have $\lim _{n \rightarrow \infty} u_{n}\left(\zeta_{0}\right)=-\infty$. This implies that we can extract a subsequence $\left\{u_{n_{k}}\left(\zeta_{0}\right)\right\}_{k \geq 1}$ such that $\lim _{k \rightarrow \infty} u_{n_{k}}\left(\zeta_{0}\right)=-\infty$. By the definition of $u(z, w)$ we see that the sequence $\left\{\left(f_{n_{k}}^{1}\left(\zeta_{0}\right), \operatorname{Re} f_{n_{k}}^{2}\left(\zeta_{0}\right)\right)\right\}_{k \geq 1}$ is not bounded. Then there are two subcases to consider: (i) There is a subsequence, without loss of generality still denoted by $\left\{f_{n_{k}}\right\}$, such that $\left|f_{n_{k}}^{1}\left(\zeta_{0}\right)\right| \rightarrow \infty$ as $k \rightarrow \infty$. Notice that $\left\{f_{n_{k}}^{1}\right\}$ is also equicontinuous; thus for $\varepsilon=1$ we can find a $\delta>0$ such that

$$
\left|f_{n_{k}}^{1}(\zeta)-f_{n_{k}}^{1}\left(\zeta_{0}\right)\right| \leq 1, \quad \forall k \geq 1,\left|\zeta-\zeta_{0}\right| \leq \delta .
$$

Since $\zeta_{j} \rightarrow \zeta_{0}$, we may choose a $j_{0}$ so that $\left|\zeta_{j_{0}}-\zeta_{0}\right| \leq \delta$. Then, for any $k \geq 1$, we get $\left|f_{n_{k}}^{1}\left(\zeta_{0}\right)-f_{n_{k}}^{1}\left(\zeta_{j_{0}}\right)\right| \leq 1$. This implies that $\left|f_{n_{k}}^{1}\left(\zeta_{j_{0}}\right)\right| \geq\left|f_{n_{k}}^{1}\left(\zeta_{0}\right)\right|-1$. It follows that $\lim _{k \rightarrow \infty}\left|f_{n_{k}}^{1}\left(\zeta_{j_{0}}\right)\right|=\infty$. Then by the trivial estimate $u(z, w) \leq$ $-a(z)$, for all $(z, w) \in D$, and the fact that $f_{n} \in H(\Delta, D)$, we conclude that $u_{n_{k}}\left(\zeta_{0}\right) \rightarrow-\infty$ as $k \rightarrow \infty$. This implies in turn that $\underline{\lim }_{n \rightarrow \infty} u_{n}\left(\zeta_{j_{0}}\right)=-\infty$ which contradicts our assumption $\zeta_{j_{0}} \in A$. (ii) The sequence $\left\{f_{n_{k}}^{1}\left(\zeta_{0}\right)\right\}$ is bounded but $\left\{\operatorname{Re} f_{n_{k}}^{2}\left(\zeta_{0}\right)\right\}$ is not. We may assume that $\operatorname{Re} f_{n_{k}}^{2}\left(\zeta_{0}\right) \rightarrow-\infty$ as $k \rightarrow \infty$. Arguing similarly to the case (i), and noting that $\left\{\operatorname{Re} f_{n_{k}}^{2}\right\}$ is also equicontinuous, we may find a $j_{0}$ such that $-\operatorname{Re} f_{n_{k}}^{2}\left(\zeta_{j}\right) \rightarrow \infty$ as $k \rightarrow \infty$ and $j \geq j_{0}$. Moreover, since $\left\{f_{n_{k}}^{1}\left(\zeta_{0}\right)\right\}$ is bounded and $\zeta_{j} \rightarrow \zeta_{0}$, the equicontinuity 
of $\left\{f_{n_{k}}^{1}\right\}$ implies that $\left\{f_{n_{k}}^{1}\left(\zeta_{j}\right)\right\}_{k, j \geq 1}$ is also bounded. Therefore we may choose a $j_{0}$ such that

$$
u_{n_{k}}\left(\zeta_{j_{0}}\right)=\operatorname{Re} f_{n_{k}}^{2}\left(\zeta_{j_{0}}\right)+(P-a)\left(f_{n_{k}}^{1}\left(\zeta_{j_{0}}\right)\right) \rightarrow-\infty \text { as } k \rightarrow \infty .
$$

This also implies that $\underline{\lim }_{n \rightarrow \infty} u_{n}\left(\zeta_{j_{0}}\right)=-\infty$, which again contradicts the assumption that $\zeta_{j_{0}} \in A$. So we see that $\zeta_{0} \in A$ and $A$ is closed as well.

Since $\Delta$ is connected, we must have $A=\Delta$. That is to say, for any $\zeta \in \Delta$, $\underline{\lim }_{n} u_{n}(\zeta)>-\infty$. Then the special form of $u$ implies again that $\left\{f_{n}^{1}(\zeta)\right\}$ and $\left\{\operatorname{Re} f_{n}^{2}(\zeta)\right\}$ are bounded for each $\zeta \in \Delta$. The Ascoli-Arsela theorem can then be applied to conclude that there exists a subsequence $\left\{f_{n_{k}}\right\}$ and holomorphic mappings $f^{1} \in H\left(\Delta, \mathbb{C}^{n}\right), f^{2} \in H(\Delta, \mathbb{C})$ such that

$f_{n_{k}}^{1} \rightarrow f^{1}, \quad \operatorname{Re} f_{n_{k}}^{2} \rightarrow \operatorname{Re} f^{2}, \quad$ uniformly on compacta of $\Delta$, as $k \rightarrow \infty$.

If for all $\zeta \in \Delta,\left\{f_{n_{k}}^{2}(\zeta)\right\}$ is unbounded, then $\left\{f_{n_{k}}(\zeta)\right\}$ is unbounded for all $\zeta \in \Delta$ as well. Now with the same arguments as in case (1) one can show that $\left\{f_{n_{k}}\right\}$ is compactly divergent. On the other hand, if there is a $\zeta_{0} \in \Delta$ such that $\left\{f_{n_{k}}^{2}\left(\zeta_{0}\right)\right\}$ is bounded, then we may assume that $f_{n_{k}}^{2}\left(\zeta_{0}\right)$ converges, after possibly extracting a subsequence. In view of (3.12), we conclude that $f_{n_{k}}^{2} \rightarrow f^{2}$ uniformly on compacta of $\Delta$. Then $f_{n_{k}} \rightarrow\left(f^{1}, f^{2}\right)$ uniformly on compacta of $\Delta$ as $k \rightarrow \infty$. This is the end of case (2). Cases (1) and (2) then complete the proof of the lemma.

\section{LOCAL GEOMETRY OF h-EXTENDIBLE DOMAINS}

By a pointed domain $(\Omega, p)$ in $\mathbb{C}^{n}$ we mean that $\Omega$ is a smooth pseudoconvex domain in $\mathbb{C}^{n}$ with $p \in b \Omega$. Let $\rho$ be a local defining function for $\Omega$ near $p$ and let the multitype $\mathscr{M}(p)=\left(m_{1}, \ldots, m_{n}\right)$ be finite, i.e., $m_{n}<\infty$. By the definition of multitype, there are (distinguished) coordinates $z=\left(z_{1}, z^{\prime}\right)$ such that $p=0$ and $\rho(z)$ can be expanded near 0 as follows:

$$
\rho(z)=\operatorname{Re} z_{1}+P\left(z^{\prime}\right)+R(z) .
$$

Here $P$ is $\left(1 / m_{2}, \ldots, 1 / m_{n}\right)$-homogeneous plurisubharmonic polynomial that contains no pluriharmonic terms, $R$ is smooth and satisfies

$$
|R(z)| \leq C\left(\sum_{i=1}^{n}\left|z_{i}\right|^{m_{i}}\right)^{\gamma},
$$

for some constant $\gamma>1$ and $C>0$. The polynomial $P$ is called the principal part of $\rho$.

Definition 4.1. We call $D=\left\{z \in \mathbb{C}^{n}: \operatorname{Re} z_{1}+P\left(z^{\prime}\right)<0\right\}$ an associated model for $(\Omega, p)$. If the pointed domain $(\Omega, p)$ has an h-extendible associated model, we say that $(\Omega, p)$ is h-extendible. A pseudoconvex domain is called h-extendible if $(\Omega, p)$ is h-extendible for all $p \in b \Omega$.

It is apparent from our definition that the property of being h-extendible is biholomorphically invariant, that is, if $(\Omega, p)$ is h-extendible and $\Phi$ is a local biholomorphism near $p$, then $(\Phi(\Omega), \Phi(p))$ is also h-extendible.

Let us first look at a few examples. 
Proposition 4.2. Let $\Omega$ be a smoothly bounded pseudoconvex domain in $\mathbb{C}^{2}$ and $p \in \partial \Omega$ a point of finite type. Then $(\Omega, p)$ is h-extendible.

This follows from Example 3.5. By using the special coordinates found in [BP2] or [YU1] and the result in Example 3.4, one easily sees that

Proposition 4.3. If $\Omega$ is a smooth, convex domain in $\mathbb{C}^{n+1}$ and $p$ a boundary point of finite type, then $(\Omega, p)$ is h-extendible.

Proposition 4.4. If $\Omega$ is a decoupled domain of finite type, then it is h-extendible.

This follows from Example 3.6. For further properties of decoupled domains, see $[\mathrm{MC}]$.

Proposition 4.5. Let $\Omega$ be a smooth pseudoconvex domain in $\mathbb{C}^{n+1}$ with $p \in \partial \Omega$ of finite type. If the Levi form at $p$ has rank greater than or equal to $n-1$, then $(\Omega, p)$ is h-extendible.

The proof of this fact is similar to that of Proposition 3.3 (cf. [BP1]).

Proposition 4.6. Let $\Omega$ be a smooth pseudoconvex domain in $\mathbb{C}^{n+1}$ with $p$ a finite type boundary point. If $(\Omega, p)$ has a homogeneous model, then it is $h$ extendible.

This is a direct consequence of Proposition 3.7 (cf. [NO]).

From Propositions 4.2-4.6 we see that the class of h-extendible domains is indeed a fairly large class of weakly pseudoconvex domains which covers many interesting domains. In fact, it will be shown in [YU4] that a pointed domain is h-extendible iff the multitype is the same as the q-types.

In order to study the boundary behavior of the Kobayashi metrics on an h-extendible domain, we need to relate its local Levi geometry to that of its model. In this connection, we have

Theorem 4.7. Let $(D, p)$ be an h-extendible pointed domain in $\mathbb{C}^{n+1}$. Then there are local holomorphic coordinates $(z, w)$ and an h-extendible model $D_{p}$ such that $p=(0,0)$ and $\bar{D} \backslash\{p\} \subset D_{p}$ near $p$.

Here and in the sequel, we always denote by $\Lambda$ the multiweight $\left(1 / m_{n}, \ldots\right.$, $\left.1 / m_{1}\right)$ with $\left(1, m_{1}, \ldots, m_{n}\right)=\mathscr{M}(p)$.

To prove Theorem 4.7 we first fix some notations.

Definition 4.8. For any integer $n \geq 1$, let $\Lambda=\left(\lambda_{1}, \ldots, \lambda_{n}\right)$ be a fixed $n$-tuple of positive numbers and $\mu>0$. We denote by $\mathscr{O}(\mu, \Lambda)$ the set of smooth functions $f$ defined near the origin of $\mathbb{C}^{n}$ such that

$$
D^{\alpha} \bar{D}^{\beta} f(0)=0 \quad \text { whenever } \quad \sum_{i=1}^{n}\left(\alpha_{i}+\beta_{i}\right) \lambda_{i} \leq \mu .
$$

Here $\alpha, \beta$ are multi-indices and $D^{\alpha}=\partial^{|\alpha|} / \partial z_{1}^{\alpha_{1}} \cdots \partial z_{n}^{\alpha_{n}}$. If $n=1$ and $\Lambda=(1)$ then we use $\mathscr{O}(\mu)$ to denote the functions vanishing to order at least $\mu$ at the origin.

Similarly let $\mathscr{H}(\mu, \Lambda)$ denote the set of polynomials $f$ such that

$$
D^{\alpha} \bar{D}^{\beta} f(0)=0 \quad \text { whenever } \quad \sum_{i=1}^{n}\left(\alpha_{i}+\beta_{i}\right) \lambda_{i} \neq \mu .
$$

Some simple properties of $\mathscr{O}(\mu, \Lambda)$ and $\mathscr{H}(\mu, \Lambda)$ are given in the following proposition, which can be easily verified by using Taylor's Theorem: 


\section{Proposition 4.9.}

(i) If $f \in \mathscr{O}(\mu, \Lambda)($ or $\mathscr{H}(\mu, \Lambda))$, then $\left(\partial f / \partial z_{k}\right)$ and $\left(\partial f / \partial \bar{z}_{k}\right)$ are in $\mathscr{O}\left(\mu-\lambda_{k}, \Lambda\right)\left(\right.$ or $\left.\mathscr{H}\left(\mu-\lambda_{i}, \Lambda\right)\right)$.

(ii) Suppose that $f_{i}, 1 \leq i \leq N$, are functions with $f_{i} \in \mathscr{O}\left(\mu_{i}, \Lambda\right)$ (or $\left.\mathscr{H}\left(\mu_{i}, \Lambda\right)\right)$. Then

$$
\prod_{i=1}^{N} f_{i} \in \mathscr{O}(\mu, \Lambda) \quad(\text { or } \mathscr{H}(\mu, \Lambda)), \quad \text { where } \mu=\sum_{i=1}^{N} \mu_{i} .
$$

(iii) If $f \in \mathscr{O}(\mu, \Lambda)$, then there are constants $\varepsilon, C>0$ such that

$$
|f(z)| \leq C\left(\sigma_{\Lambda}(z)\right)^{\mu+\varepsilon} \quad \forall z \text { in a small neighborhood of } 0 .
$$

The following two lemmas are higher dimensional generalizations of similar facts in [F-S].

Lemma 4.10. Let $D$ be any pseudoconvex domain in $\mathbb{C}^{n+1}$ with smooth boundary and $p$ a boundary point. Let $\Lambda \in \mathscr{W}_{n}$ be any multiweight. Then there are local holomorphic coordinates $(z, w)$ such that $p=0$ and near $p$ the domain $D$ can be described as

$$
\begin{aligned}
& D(z, w)=\{(z, w): \operatorname{Re} w+\sum_{i=1}^{M} P_{i}(z)+R_{1}(z)+R_{2}(\operatorname{Im} w) \\
&\left.+(\operatorname{Im} w) \sum_{i=1}^{N} Q_{i}(z)+(\operatorname{Im} w) R(z)<0\right\} .
\end{aligned}
$$

Here $P_{i}, Q_{i}$ are real $\Lambda$-homogeneous polynomials with weight $\alpha_{i}, \beta_{i}$ respectively, containing no pluriharmonic terms, such that

$$
0<\alpha_{1}<\cdots<\alpha_{M} \leq 1 \quad \text { and } 0<\beta_{1}<\cdots<\beta_{N} \leq \frac{1}{2} .
$$

The functions $R_{1}, R_{2}, R$ are real smooth functions such that $R_{1}, R$ are independent of $w$ satisfying the following conditions:

$$
R_{1} \in \mathscr{O}(1, \Lambda), R \in \mathscr{O}(1 / 2, \Lambda), R_{2} \in \mathscr{O}(2) .
$$

Proof of Lemma 4.10. Let $r$ be a local smooth defining function for $D$ at $p$. By translation and rotation we may assume that $p=0$ and using Taylor's formula we can expand $r$ near 0 as

$$
\begin{aligned}
r(z, w)= & \operatorname{Re} w+\sum_{i=1}^{M_{1}} P_{i}(z)+R_{1}(z)+R_{2}(\operatorname{Im} w) \\
& +(\operatorname{Im} w) \sum_{i=1}^{N_{1}} Q_{i}(z)+(\operatorname{Im} w) R(z) .
\end{aligned}
$$

Here $P_{i}, Q_{i}, R_{1}, R_{2}$ and $R$ have all the properties required except that $P_{i}, Q_{i}$ may contain p.h.(pluriharmonic) monomials; moreover, $M_{1}, N_{1}$ are the numbers of polynomials in the expansion that have weights less than or equal to $1,1 / 2$ w.r.t. $\Lambda$ respectively. Hence it remains to remove all p.h. monomials from the $P_{i}$ 's and $Q_{i}$ 's. 
Recall that a real smooth function on $\mathbb{C}^{n}$ is p.h. if and only if it is the real part (or the imaginary part) of a holomorphic function on $\mathbb{C}^{n}$ (cf. [KR1]); a real monomial is p.h. if and only if it can be written as $\operatorname{Re}\left(a_{J} z^{J}\right)\left(\right.$ or $\left.\operatorname{Im}\left(a_{J} z^{J}\right)\right)$, where $J$ is a multi-index. Now we begin to remove p.h. monomials from the $P_{i}$ 's by induction. Assume that $1 \leq j \leq M$ is the least index for which $P_{i}$ contains a p.h. term. Denote the p.h. term by $\operatorname{Re} h(z)$. Then, after introducing the local holomorphic coordinate change $\tilde{w}=w+h(z), \tilde{z}=z$, we can describe the domain near 0 as

$$
\begin{aligned}
& D(\tilde{z}, \tilde{w})=\left\{\operatorname{Re} \tilde{w}+\sum_{i \neq j} P_{i}(\tilde{z})+\tilde{P}_{j}(\tilde{z})+R_{1}(\tilde{z})+R_{2}(\operatorname{Im}(\tilde{w}-h(\tilde{z})))\right. \\
& +(\operatorname{Im} \tilde{w}) \sum_{i=1}^{N_{1}} Q_{i}(\tilde{z})-(\operatorname{Im} h(\tilde{z})) \sum_{i=1}^{N_{1}} Q_{i}(\tilde{z})+(\operatorname{Im} \tilde{w}) R(\tilde{z}) \\
& -(\operatorname{Im} h(\tilde{z})) R(\tilde{z})<0\} .
\end{aligned}
$$

Collecting terms, we see that $D$ has the same form as above except that now $P_{1}, \ldots, P_{j}$ are all without p.h. terms. Continuing like this we see that we can remove all the p.h. terms from the $P_{i}$ 's.

Next we remove the p.h. terms from the $Q_{i}$ 's without reintroducing p.h. terms into the $P_{i}$ 's. This can be done also by induction, as explained below.

Let $j$ be the least index for which $Q_{j}$ contains p.h. terms. Write $Q_{j}(z)=$ $\operatorname{Im} h_{j}(z)+\tilde{Q}_{j}(z)$, where $\tilde{Q}_{j}$ contains no p.h. terms or $\tilde{Q}_{j} \equiv 0$. It is convenient now to describe the boundary of $D$ near 0 as given by

$$
\operatorname{Re} w=\sum_{i=1}^{M_{1}} P_{i}(z)+R_{1}(z)+R_{2}(\operatorname{Im} w)+(\operatorname{Im} w) \sum_{i=1}^{N_{1}} Q_{i}(z)+(\operatorname{Im} w) R(z)
$$

To get rid of $h_{j}$, multiply both sides of $(4.5)$ by $1+\operatorname{Re}\left(h_{j}(z)\right)$. Let $\tilde{w}=$ $w\left(1+h_{j}(z)\right)$. Since $\operatorname{Re} \tilde{w}=\operatorname{Re} w+\operatorname{Re} w \operatorname{Re} h_{j}(z)-\operatorname{Im} w \operatorname{Im} h_{j}(z)$, we can now swallow up the p.h. term from $Q_{j}$ into $\operatorname{Re} \tilde{w}$. We only need to check that we do not at the same time reintroduce p.h. terms elsewhere.

So we obtain:

$$
\begin{aligned}
\operatorname{Re} \tilde{w}= & \sum_{i=1}^{M_{1}} P_{i}+R_{1}(z)+R_{2}(\operatorname{Im} w)+(\operatorname{Im} w) \sum_{i=1}^{j} Q_{i}(z) \\
& +\sum_{i=j+1}^{N_{1}} Q_{i}(z)+(\operatorname{Im} w) R(z) .
\end{aligned}
$$

Here and in the following, by abuse of notation, we use $R$ 's, $P$ 's and $Q$ 's to denote different functions with the same properties listed before. We still need to replace $w$ by $\tilde{w}$ everywhere.

Since $w=\tilde{w} /\left(1+h_{j}(z)\right)=\tilde{w}\left(1-h_{j}(z)+\sigma_{j+1}(z)\right)$, where $\sigma_{j+1}(z)$ is a smooth function such that $\sigma_{j+1} \in \mathscr{O}\left(\beta_{j}, \Lambda\right)$, we have

$$
\begin{aligned}
\operatorname{Im} w & =\operatorname{Im} \tilde{w}+\operatorname{Re} \tilde{w} \operatorname{Im}\left(\sigma_{j+1}(z)-h_{j}(z)\right)+\operatorname{Im} \tilde{w} \operatorname{Re}\left(\sigma_{j+1}-h_{j}(z)\right) \\
& =\operatorname{Im} \tilde{w}\left(1+\sigma_{j}(z)\right)+\operatorname{Re} \tilde{w} \sigma_{j}^{\prime}(z) .
\end{aligned}
$$


Here, $\sigma_{j}, \sigma_{j}^{\prime}$ are smooth functions in $\mathscr{O}\left(\beta_{j}, \Lambda\right)$. Substituting (4.6) into (4.5) and collecting similar terms, we can write

$$
\begin{aligned}
\operatorname{Re} \tilde{w}= & \sum_{i=1}^{M_{1}} P_{i}(z)+R_{1}(z)+R_{2}(\operatorname{Im} \tilde{w})+(\operatorname{Im} \tilde{w}) \sum_{i=1}^{j} Q_{i}(z) \\
& +\sum_{i=j+1}^{N_{1}} Q_{i}^{\prime}(z)+(\operatorname{Re} \tilde{w}) \sigma(z)+(\operatorname{Im} \tilde{w}) R(z) .
\end{aligned}
$$

Here $\sigma(z)$ is a smooth function with positive weight. Then move the term $\operatorname{Re} \tilde{w} \sigma(z)$ on the right side to the left, and divide out the term $1-\sigma(z)$, noting that $\frac{1}{1-\sigma(z)}=1+\sigma^{\prime}(z)$, where $\sigma^{\prime}(z)$ has positive weight. We obtain

$$
\begin{aligned}
\operatorname{Re} \tilde{w}= & \sum_{i=1}^{M_{1}} P_{i}(z)\left(1+\sigma^{\prime}(z)\right)+R_{1}^{\prime}(z)+R_{2}^{\prime}(\operatorname{Im} \tilde{w})+(\operatorname{Im} \tilde{w}) \sum_{i=1}^{j} Q_{i}(z) \\
& +\sum_{i=j+1}^{N_{1}} Q_{i}^{\prime \prime}(z)+\operatorname{Im} \tilde{w} R^{\prime}(z) .
\end{aligned}
$$

Expand $\sigma^{\prime}(z)$ at 0 and observe that the multiplication of $P_{i}$ with any polynomial still contains no p.h. terms. We see that we have removed the p.h. terms from $Q_{j}$ without reintroducing p.h. terms into the $P_{i}$ 's. Continuing in this way a finite number of steps, we will remove all the p.h. terms from the $Q_{i}$ 's. This proves the lemma.

Lemma 4.11. Let $(D, p)$ be a pointed domain in $\mathbb{C}^{n+1}$. Suppose that the multitype of $p$ is $\left(1, m_{1}, \ldots, m_{n}\right)$ with $m_{n}<\infty$ and let $\Lambda=\left(1 / m_{n}, \ldots, 1 / m_{1}\right)$. Then there are local holomorphic coordinates $(z, w)$ in which $p=0$ and $D$ can be described near $p$ as follows:

$$
D(z, w)=\left\{\operatorname{Re} w+P(z)+R_{1}(z)+R_{2}(\operatorname{Im} w)+(\operatorname{Im} w) R(z)<0\right\} .
$$

Here $P$ is a $\Lambda$-homogeneous p.s.h. real-valued polynomial containing no p.h. monomials, $R_{1} \in \mathscr{O}(1, \Lambda), R \in \mathscr{O}\left(\frac{1}{2}, \Lambda\right)$ and $R_{2} \in \mathscr{O}(2)$.

Proof. First apply Lemma 4.10 with $\Lambda$ given as above to find local holomorphic coordinates $(z, w)$ in which $p=0$ and near $p, D$ is given by a smooth defining function

$$
\begin{aligned}
r(z, w)= & \operatorname{Re} w+\sum_{i=1}^{M} P_{i}(z)+R_{1}(z)+R_{2}(\operatorname{Im} w)+(\operatorname{Im} w) \sum_{i=1}^{N} Q_{i}(z) \\
& +(\operatorname{Im} w) R(z) .
\end{aligned}
$$

Here $P_{i}, Q_{i}$ are real $\Lambda$-homogeneous polynomials with weight $\alpha_{i}, \beta_{i}$ respectively, where $\alpha_{i}, \beta_{i}$ satisfy condition (4.2). Moreover, $P_{i}, Q_{i}$ do not contain any p.h. monomials. Now since 0 has multitype $\left(1, m_{1}, \ldots, m_{n}\right)$ we must have $P_{i} \equiv 0$ for $1 \leq i<M$ and $P_{M} \not \equiv 0$. We will denote $P_{M}$ by $P$ for simplicity. Thus the defining function $r$ can be written as

$$
r(z, w)=\operatorname{Re} w+P(z)+R_{1}(z)+R_{2}(\operatorname{Im} w)+(\operatorname{Im} w) \sum_{i=1}^{N} Q_{i}(z)+(\operatorname{Im} w) R(z) .
$$


Next we will use the pseudoconvexity of the domain to (i) show that $P$ is p.s.h. and (ii) eliminate the $Q_{i}$ 's. In order to do so, let us look at the Levi form of $r$. By definition, $D$ is pseudoconvex near 0 if and only if (4.9)

$$
\sum_{j, k=1}^{n} r_{j k}(z, w) X_{j} \bar{X}_{k}+2 \operatorname{Re}\left(\sum_{j=1}^{n} r_{j w}(z, w) X_{j} \bar{X}_{n+1}\right)+r_{w w}(z, w)\left|X_{n+1}\right|^{2} \geq 0,
$$

for all $(z, w) \in \partial D$ near 0 and $X=\left(X_{1}, \ldots, X_{n+1}\right)$ with

$$
\sum_{j=1}^{n} r_{j}(z, w) X_{j}+r_{w}(z, w) X_{n+1}=0 .
$$

Here and in the following, $r_{j k}=\partial^{2} r / \partial z_{j} \bar{\partial} z_{k}, r_{j}=\partial r / \partial z_{j}$ and $r_{w}=\partial r / \partial w$; $r_{w \bar{w}}, r_{j \bar{w}}$ are defined in a similar way. Substituting (4.10) into (4.9), we obtain

$$
\begin{aligned}
\sum_{j, k=1}^{n} r_{j \bar{k}}(z, w)\left|r_{w}(z, w)\right|^{2} X_{j} \bar{X}_{k} & -2 \operatorname{Re} \sum_{j, k=1}^{n} r_{j w}(z, w) r_{w}(z, w) r_{\bar{k}}(z, w) X_{j} \bar{X}_{k} \\
& +r_{w w}(z, w)\left|\sum_{j=1}^{n} r_{j}(z, w) X_{j}\right|^{2} \geq 0 .
\end{aligned}
$$

The inequality (4.11) holds for all $(z, w) \in \partial D$ near 0 and all $X \in \mathbb{C}^{n}$. Actually, if we set $u=\operatorname{Re} w, v=\operatorname{Im} w$, since the boundary of $D$ near 0 can be described as $u=f(z, v)$ with $f$ a real-valued smooth function, then the left-hand side of (4.11) is a smooth function of $(z, v)$ near 0 and (4.11) holds for all $(z, v) \in \mathbb{C}^{n} \times \mathbb{R}$ near 0 . If we write out $r_{j k}$ and so on using (4.9), then we get

$$
\begin{aligned}
& r_{j k}(z, w)=P_{j k}(z)+R_{1 j k}(z)+R_{2 j k}(v)+v\left(\sum_{i=1}^{N} Q_{i j k}(z)+R_{j k}(v)\right), \\
& r_{w}(z, w)=\frac{1}{2}+R_{2 w}(v)+\frac{1}{2 \pi}\left(\sum_{i=1}^{N} Q_{i}(z)+R(z)\right), \\
& r_{j w}(z, w)=R_{2 j w}(v)-\frac{1}{2 \pi}\left(\sum_{i=1}^{N} Q_{i j}(z)+R_{j}(z)\right), \\
& r_{j}(z, w)=P_{j}(z)+R_{1 j}(z)+R_{2 j}(v)+v\left(\sum_{i=1}^{N} Q_{i j}(z)+R_{j}(z)\right) .
\end{aligned}
$$

It follows from the conditions on $P, R$ 's, $Q_{i}$ 's and Proposition 4.9 that

$$
\begin{array}{ll} 
& P_{j k} \in \mathscr{H}\left(1-\lambda_{j}-\lambda_{k}, \Lambda\right), \quad Q_{i j k} \in \mathscr{H}\left(\beta_{i}-\lambda_{j}-\lambda_{k}, \Lambda\right), \\
& R_{1 j k} \in \mathscr{O}\left(1-\lambda_{j}-\lambda_{k}, \Lambda\right), \quad R_{2 j k} \in \mathscr{O}(2), R_{2 j} \in \mathscr{O}(2), \\
& P_{j} \in \mathscr{H}\left(1-\lambda_{j}, \Lambda\right), \quad R_{1 j} \in \mathscr{O}\left(1-\lambda_{j}\right), Q_{i j} \in \mathscr{H}\left(\beta_{i}-\lambda_{j}, \Lambda\right), \\
& R_{j k} \in \mathscr{O}\left(1 / 2-\lambda_{j}-\lambda_{k}, \Lambda\right), \quad R_{j} \in \mathscr{O}\left(1 / 2-\lambda_{j}, \Lambda\right), \\
& R_{2 w} \in \mathscr{O}(1), \quad R_{2 j w} \in \mathscr{O}(1) .
\end{array}
$$


Now let us make the following rescaling in (4.11) and (4.12):

$$
z \rightarrow \pi_{t}(z), \quad w \rightarrow t w, \quad X \rightarrow \pi_{t}(X),
$$

for any $(z, w)$ and $t>0$ sufficiently small. Here $\pi_{t}$ was defined by (3.1) in $\S 3$. Divide by $t$ on both sides of (4.11) after the rescaling and use the weight estimates (4.12), we see that as $t \rightarrow 0^{+}$,

$$
\text { the first sum in }(4.11) \longrightarrow \sum_{j, k=1}^{n} P_{j \bar{k}}(z) X_{j} \bar{X}_{k},
$$

the second and the third sum in (4.11) $\longrightarrow 0$.

This implies, for all $z \in \mathbb{C}^{n}, X \in \mathbb{C}^{n}$, that $\sum_{j, k=1}^{n} P_{j \bar{k}}(z) X_{j} \bar{X}_{k} \geq 0$, which means exactly that $P$ is plurisubharmonic.

Next we show that all $Q_{i}$ 's have to vanish identically by contradiction. So assume, without loss of generality, that $Q_{1} \not \equiv 0$. By our hypothesis $Q_{1}$ is a real $\Lambda$-homogeneous polynomial with lowest weight $\beta_{1} \in\left(0, \frac{1}{2}\right]$ among the $Q_{i}$ 's and is not p.h. We make another rescaling in (4.11):

$$
z \rightarrow \pi_{t}(z), \quad v \rightarrow t^{1 / 2} v, \quad X \rightarrow \pi_{t}(X) .
$$

Again, substituting (4.14) into (4.11) and dividing out $t^{\frac{1}{2}+\beta_{1}}$, and using the estimates (4.12) and (4.13), together with the assumption that $\beta_{1} \in\left(0, \frac{1}{2}\right]$, we claim that as $t \rightarrow 0^{+}$

the first sum in $(4.11) \longrightarrow \frac{1}{4} \sum_{j, k=1}^{n} \delta_{\frac{1}{2} \beta_{1}}\left(P_{j \bar{k}}(z)+v Q_{1 j \bar{k}}(z)\right) X_{j} \bar{X}_{k}$,

the second and the third term in $(4.11) \longrightarrow 0$.

Here $\delta_{\frac{1}{2} \beta_{1}}=0$ if $\beta_{1} \neq \frac{1}{2}$ and $=1$ if $\beta_{1}=\frac{1}{2}$. Therefore we obtain from (4.11) that

$$
\sum_{j, k=1}^{n}\left(\delta_{\frac{1}{2} \beta_{1}} P_{j \bar{k}}(z)+v Q_{1 j \bar{k}}(z)\right) X_{j} \bar{X}_{k} \geq 0,
$$

for all $z \in \mathbb{C}^{n}, X \in \mathbb{C}^{n}, v \in \mathbb{R}$. This is impossible for $Q_{1}$ not p.h. This contradiction finishes the proof of the lemma.

Finally we are ready to prove Theorem 4.7 . So assume that $(D, p)$ is an $\mathrm{h}$ extendible pointed pseudoconvex domain in $\mathbb{C}^{n+1}$. Apply Lemma 4.11 to find local holomorphic coordinates $(z, w)$ in which $p=0$ and near $p$ the domain $D$ is defined by

$$
r(z, w)=u+P(z)-R_{1}(z)-R_{2}(v)-v R(z) .
$$

Here $P, R_{i}, R_{2}, R$ satisfy the conditions in Lemma 4.11 and $w=u+i v$. Let $2 a(z)$ be a bumping function for $P$. This implies that $a(z)$ is a bumping function for $P(z)-a(z)$. Consider the function $r_{K}(z, w)=\operatorname{Re}\left(w+K w^{2}\right)+$ $P(z)-a(z)$. From (4.15) we get

$$
u^{2} \leq 4\left[r^{2}(z, w)+P^{2}(z)+R_{1}^{2}(z)+R_{2}^{2}(v)+v^{2} R^{2}(z)\right] .
$$


Substituting (4.15) and (4.16) in $r_{K}$, we get

$$
\begin{aligned}
r_{K}(z, w)= & u+K\left(u^{2}-v^{2}\right)+P(z)-a(z) \\
\leq & r(z, w)-P(z)+R_{1}(z)+R_{2}(v)+v R(z)+4 K\left(r^{2}(z, w)+P^{2}(z)\right. \\
& \left.+R_{1}^{2}(z)+R_{2}^{2}(v)+v^{2} R^{2}(z)\right)-K v^{2}+P(z)-a(z) \\
\leq & r(z, w)+4 K r^{2}(z, w)+R_{1}(z)+R_{2}(v)+v^{2}+R^{2}+4 K\left(P^{2}(z)\right. \\
& \left.+R_{1}^{2}(z)+R_{2}^{2}(v)+v^{2} R^{2}(z)\right)-K v^{2}-a(z) \\
= & r(z, w)+4 K r^{2}(z, w) \\
& +\left[R_{1}(z)+R^{2}(z)+4 K P^{2}(z)+4 K R_{1}^{2}(z)-a(z)\right] \\
& +v^{2}\left[1+\frac{R_{2}(v)}{v^{2}}+4 K R^{2}+4 K \frac{R_{2}^{2}(v)}{v^{2}}-K\right] \\
= & r(z, w)+4 K r^{2}(z, w)+I+v^{2} I I .
\end{aligned}
$$

Here $I, I I$ are defined by the obvious expressions. By checking the weights of the terms in $I$ and $I I$ using Proposition 4.9, we can easily obtain the following estimates for $(z, v)$ near 0 :

$$
\begin{aligned}
& R_{1}(z) \lesssim(\sigma(z))^{1+\varepsilon}, \quad R^{2}(z) \lesssim(\sigma(z))^{1+\varepsilon}, \\
& 4 K P^{2}(z) \lesssim 4 K \sigma^{2}(z), \quad 4 K R_{1}^{2}(z) \lesssim 4 K \sigma^{2}(z), \\
& R_{2}(v) \lesssim v^{2},
\end{aligned}
$$

for some $\varepsilon>0$. Recalling that $a(z)$ satisfies (3.3), we thus obtain that

$$
\begin{aligned}
& I \lesssim \sigma(z)\left[2 \sigma(z)^{\varepsilon}+8 K \sigma(z)-1\right], \\
& I I \lesssim 1+K\left(4 \sigma(z)^{1+\varepsilon}+v^{2}-1\right) .
\end{aligned}
$$

Now, if we first choose a $K>4$, then for $|(z, w)|>0$ small enough we will have $I \leq-\frac{1}{2} \sigma(z), I I<0$ and $4 K r^{2}(z, w)<\frac{1}{2}|r(z, w)|$. It follows from (4.17) that, for such a choice of $K$, there is a small neighborhood $U$ of 0 in $\mathbb{C}^{n+1}$ such that

$$
\begin{aligned}
r_{K}(z, w) & <r(z, w)+4 K r^{2}(z, w)-\sigma(z) \quad \forall(z, w) \in U \\
& <\frac{1}{2}(r(z, w)-\sigma(z)), \quad \forall(z, w) \in U \cap D .
\end{aligned}
$$

Consequently, if we introduce another local holomorphic coordinate change

$$
\tilde{w}=w+K w^{2}, \quad \tilde{z}=z,
$$

and set $D_{p}=\{(\tilde{z}, \tilde{w}): \operatorname{Re} \tilde{w}+P(\tilde{z})-a(\tilde{z})<0\}$, then for a small neighborhood $U^{\prime}$ of 0 we will have $\bar{D} \cap U^{\prime} \backslash\{0\} \subset D_{p}$. Observe that in these new coordinates $D$ still has the same form as in (4.7). Further, $D_{p}$ is an h-extendible model in $\mathbb{C}^{n+1}$ by our choice of the bumping function $a$. This is exactly what we seek. Hence the proof is complete.

Remark. It can be proved by using Theorem 4.7 and the proof of Theorem 3.11 that every boundary point of an h-extendible domain is a peak point 
[YU4]. Consequently, any h-extendible domain is complete with respect to the Carathéodory metric as well as to the Kobayashi metrics (cf. [GR], [YU5]).

\section{WEIGHTED BOUNDARY LIMITS OF THE KOBAYASHI METRICS}

We are now ready to prove the main theorem stated in $\S 1$. The idea is to blow up the domain using a rescaling argument. Then the boundary problem is converted to an interior problem. This idea has been very useful in solving many problems (cf. [BP1], [BP2], [FR], [PI], [KI]).

First we prove the following simplified local version of the main theorem:

Theorem 5.1. Let $D$ be an h-extendible pseudoconvex domain in $\mathbb{C}^{n+1}(\tilde{z}, \tilde{w})$ and 0 a boundary point with multitype $\left(1, m_{1}, \ldots, m_{n}\right)$. Suppose that, near $0, D$ has a smooth defining function $r$ of the form:

$$
r(\tilde{z}, \tilde{w})=\operatorname{Re} \tilde{w}+P(\tilde{z})+R_{1}(\tilde{z})+R_{2}(\operatorname{Im} \tilde{w})+(\operatorname{Im} \tilde{w}) R(\tilde{z}),
$$

where $P$ is a $\Lambda=\left(\lambda_{1}, \ldots, \lambda_{n}\right)=:\left(1 / m_{n}, \ldots, 1 / m_{1}\right)$-homogeneous real valued p.s.h. polynomial which contains no p.h. monomials and $R_{1} \in \mathscr{O}(1, \Lambda), R_{2} \in$ $\mathscr{O}(2), R \in \mathscr{O}(1 / 2, \Lambda)$. Let $\Gamma$ be a nontangential cone in $D$ with vertex at 0 . Then there is a small neighborhood $U$ of 0 such that for any $\mathbb{C}^{n+1}$-valued function $X(\xi)$ on $U$ which is continuous at 0 , we have

$$
\lim _{D \cap \Gamma \cap U \ni \xi \rightarrow 0} F_{D \cap U}\left(\xi,\left(\Pi_{|r(\xi)|}\right)_{*} X(\xi)\right)=F_{D_{0}}(0, X(0)) .
$$

Here $D_{0}=\{(z, w): \operatorname{Re} w+P(z)<1\}$ and

$$
\Pi_{t}(\xi)=\left(t^{1 / m_{n}} \xi_{1}, \ldots, t^{1 / m_{1}} \xi_{n}, t \xi_{n+1}\right), \quad \forall t>0 .
$$

Proof. Let $U^{\prime}$ be a neighborhood of 0 on which $D$ is defined by the defining function in (5.1). For any $\xi=\left(\xi^{\prime}, \xi_{0}\right) \in D \cap U^{\prime}$, where $\xi^{\prime} \in \mathbb{C}^{n}$ and $\xi_{0} \in \mathbb{C}$, set $t=|r(\xi)|$. First we observe that there is a neighborhood $U$ of 0 such that

$$
\hat{\xi}=: \Pi_{t^{-1}}(\xi) \text { is bounded on } D \cap U \cap \Gamma \text {. }
$$

More precisely, if we write $\hat{\xi}=\left(\hat{\xi}^{\prime}, \hat{\xi}_{0}\right)$, we will have

$$
\lim _{D \cap \Gamma \cap U \ni \xi \rightarrow 0} \hat{\xi}^{\prime}=0 \text { and }|\hat{\xi}| \lesssim 1 \text { on } D \cap \Gamma \cap U .
$$

To see this, note that for $\xi \in \Gamma \cap D$ near 0 ,

$$
|\xi| \lesssim\left|\operatorname{Re} \xi_{0}\right| \approx t=|r(\xi)| .
$$

Here the constants in the estimates depend only on the aperture of $\Gamma$ and on the neighborhood. Thus if $\xi \in D \cap \Gamma$ and $|\xi|$ is small, then we have

$$
\begin{aligned}
&\left|\hat{\xi}^{\prime}\right| \lesssim \sum_{i=1}^{n} t^{-\lambda_{i}}\left|\xi_{i}\right| \lesssim \sum_{i=1}^{n} t^{-\lambda_{i}} t \quad \text { by }(5.5) \\
& \longrightarrow 0 \quad \text { since } \lambda_{i}<1 \text { for } 1 \leq i \leq n . \\
&\left|\hat{\xi}_{0}\right| \lesssim t^{-1}\left|\xi_{0}\right| \lesssim t^{-1}|\xi| \lesssim 1 \quad \text { by }(5.5) .
\end{aligned}
$$

This proves (5.4) with a possibly smaller neighborhood $U$.

Now, for any $\xi \in D \cap \Gamma \cap U$, consider the following scaling:

$$
\Phi_{\xi}: \quad(\tilde{z}, \tilde{w})=\Pi_{t}(z, w)+\xi=\left(\pi_{t}(z)+\xi^{\prime}, t w+\xi_{0}\right) .
$$


Setting $r_{\xi}(z, w)=t^{-1} r\left(\Phi_{\xi}(z, w)\right)$ and $r_{0}(z, w)=\operatorname{Re} w-1+P(z)$, we show that

$$
\lim _{D \cap \Gamma \xi \rightarrow 0} r_{\xi}(z, w)=r_{0}(z, w) \quad \text { in the } C^{0} \text {-topology. }
$$

Here and in the sequel, by $C^{0}$-topology we mean uniformly on compact subsets.

To prove (5.7), let us write out $r_{\xi}$ using (5.1) in the following way:

$$
\begin{aligned}
r_{\xi}(z, w)= & t^{-1}\left(t \operatorname{Re} w+\operatorname{Re} \xi_{0}+P\left(\pi_{t}(z)+\xi^{\prime}\right)+R_{1}\left(\pi_{t}(z)+\xi^{\prime}\right)\right. \\
& \left.+R_{2}\left(t w+\xi_{0}\right)+\left(t w+\xi_{0}\right) R\left(\pi_{t}(z)+\xi^{\prime}\right)\right) \\
= & \operatorname{Re} w+t^{-1} r\left(\xi^{\prime}\right)+t^{-1}\left[P\left(\pi_{t}(z)+\xi^{\prime}\right)-P\left(\xi^{\prime}\right)\right] \\
& +t^{-1}\left[R_{1}\left(\pi_{t}(z)+\xi^{\prime}\right)-R_{1}\left(\xi^{\prime}\right)\right]+t^{-1}\left[R_{2}\left(t w+\xi_{0}\right)-R_{2}\left(\xi_{0}\right)\right] \\
& +t^{-1}\left[\left(t w+\xi_{0}\right) R\left(\pi_{t}(z)+\xi^{\prime}\right)-\xi_{0} R\left(\xi^{\prime}\right)\right] \\
= & \operatorname{Re} w-1+A+B+C+D .
\end{aligned}
$$

Here $A, B, C, D$ are defined in an obvious way. Therefore, to prove (5.7), it remains to check that, as $\xi \rightarrow 0$ in $D \cap \Gamma$

$$
A \rightarrow P(z), \quad B, C, D \rightarrow 0 \quad \text { in the } C^{0} \text {-topology. }
$$

First of all, since $P$ is a $\Lambda$-homogeneous polynomial, we have

$$
\begin{aligned}
A & =t^{-1}\left[P\left(\pi_{t}(z)+\xi^{\prime}\right)-P\left(\xi^{\prime}\right)\right]=t^{-1}\left[P\left(\pi_{t}(z)+\pi_{t}\left(\hat{\xi}^{\prime}\right)\right)-P\left(\pi_{t}\left(\hat{\xi}^{\prime}\right)\right)\right] \\
& =t^{-1}\left[P\left(\pi_{t}\left(z+\hat{\xi}^{\prime}\right)\right)-P\left(\pi_{t}\left(\hat{\xi}^{\prime}\right)\right)\right]=P\left(z+\hat{\xi}^{\prime}\right)-P\left(\hat{\xi}^{\prime}\right)=: P(z)+P_{1}\left(z ; \hat{\xi}^{\prime}\right) .
\end{aligned}
$$

Here $P_{1}\left(z ; \hat{\xi}^{\prime}\right)$ is a polynomial in which each monomial contains at least one factor from $\hat{\xi}^{\prime}$ and one factor from $z$. Hence from (5.4) it is easy to see that $A \rightarrow P(z)$ in the $C^{0}$-topology as $\xi \rightarrow 0$ in $D \cap \Gamma \cap U$.

Next, let us consider the term $B$. Since $R_{1} \in \mathscr{O}(1, \Lambda)$, using the same idea above we get

$$
\begin{aligned}
|B| & =t^{-1}\left|R_{1}\left(\pi_{t}(z)+\pi_{t}\left(\hat{\xi}^{\prime}\right)\right)-R_{1}\left(\pi_{t}\left(\hat{\xi}^{\prime}\right)\right)\right| \\
& \lesssim t^{-1}\left(\sigma\left(\pi_{t}\left(z+\hat{\xi}^{\prime}\right)\right)\right)^{1+\varepsilon} \quad \text { by Proposition } 4.9 \\
& \lesssim t^{\varepsilon}\left(\sigma\left(z+\hat{\xi}^{\prime}\right)\right)^{1+\varepsilon} \longrightarrow 0, \quad \text { in the } C^{0} \text {-topology as } D \cap \Gamma \ni \xi \rightarrow 0 \text {, by }(5.4) .
\end{aligned}
$$

Similarly, one can easily estimate that $C, D \rightarrow 0$ in the $C^{0}$-topology. This finishes the proof of (5.7). Note that if $r$ is $C^{\infty}$ smooth, then the convergence in (5.7) can be proved to hold in the $C^{\infty}$-topology.

Now let us define

$$
\begin{aligned}
& D_{\xi}=\left\{(z, w) \in \Phi_{\xi}^{-1}(U): r_{\xi}(z, w)<0\right\} \quad \text { and } \\
& D_{0}=\left\{(z, w) \in \mathbb{C}^{n+1}: r_{0}(z, w)<0\right\} .
\end{aligned}
$$

Then (5.7) implies that (cf. (2.1))

$$
\lim _{D \cap \Gamma \xi \rightarrow 0} D_{\xi}=D_{0}
$$

On the other hand, from Theorem 4.7 and its proof, there is an h-extendible model $D_{p}=\{(\tilde{z}, \tilde{w}): s(\tilde{z}, \tilde{w})=: \operatorname{Re} \tilde{w}+Q(\tilde{z})<0\}$ such that, after shrinking $U$ if necessary, we have $\bar{D} \cap U \backslash\{0)\} \subset D_{p} \cap U$. We may assume that $U \subset$ 
$U_{0}=\{(\tilde{z}, \tilde{w}): \sigma(\tilde{z}) \leq 1,|\tilde{w}| \leq 1\}$. Consider the rescaling of $D_{p} \cap U$ by the rescaling map $\Phi_{\xi}$ given by (5.6). We claim that there is a constant $c$ such that, for all $(\tilde{z}, \tilde{w}) \in U$ and $\xi \in D \cap \Gamma \cap U$ :

$$
Q\left(z+\pi_{t^{-1}}\left(\xi^{\prime}\right)\right) \geq Q(z)-c \text { for all } z \text { with } \tilde{z}=\pi_{t}(z)+\xi^{\prime} .
$$

To see this, note that for $(\tilde{z}, \tilde{w}) \in U_{0}$, we have

$$
\sigma\left(\pi_{t}(z)+\xi^{\prime}\right) \leq 1 .
$$

It follows from the following simple inequality:

$$
\sigma(x+y) \leq 2^{m}(\sigma(x)+\sigma(y)), \quad \forall x, y \in \mathbb{C}^{n},
$$

where $m$ is some positive constant, that $\sigma(x-y) \geq 2^{-m} \sigma(x)-\sigma(y)$. Putting $x=\pi_{t}(z), y=\xi^{\prime}$, we get

$$
\begin{aligned}
\sigma\left(\pi_{t}(z)+\xi^{\prime}\right) & \geq 2^{-m} \sigma\left(\pi_{t}(z)\right)-\sigma\left(\xi^{\prime}\right) \\
& \geq 2^{-m} t \sigma(z)-1 \quad \text { since } \xi \in U_{0} .
\end{aligned}
$$

From (5.10) and (5.11) we obtain that $t \leq \frac{2^{m+1}}{\sigma(z)}$, if $z \neq 0$. On the other hand, by the definition of $t$ and (5.5),

$$
\left|\pi_{t^{-1}} \xi^{\prime}\right| \leq \sum_{i=1}^{n} t^{-\lambda_{i}}\left|\xi_{i}\right| \leq \sum_{i=1}^{n} t^{-\lambda_{i}} t \leq n t^{1-\lambda_{n}}
$$

It follows from (5.11) and (5.12) that, for $|z|$ large, we have

$$
\left|\pi_{t^{-1}} \xi^{\prime}\right| \leq \frac{n 2^{m+1}}{(1+\sigma(z))^{1-\lambda_{n}}} .
$$

Then applying Lemma 3.9, we obtain (5.9). Combining (5.9) with (5.5), we finally get

$$
t^{-1} s\left(\Phi_{\xi}(z, w)\right) \geq \operatorname{Re} w+Q(z)-c^{\prime}, \quad \forall(z, w) \in \Phi_{\xi}^{-1}(U) \text { and } \xi \in D \cap \Gamma \cap U .
$$

Here $c^{\prime}$ is another positive constant. Set $\Omega=\left\{(z, w): \operatorname{Re} w+Q(z)-c^{\prime}<0\right\}$. It follows from the estimates above that

$$
D_{\xi} \subset \Omega, \quad \forall \xi \in D \cap \Gamma \cap U .
$$

Since the domain $\Omega$ is an h-extendible model and thus taut by Theorem 3.13, combining (5.8) and (5.13) we see that all conditions in Theorem 2.1 are satisfied. Therefore we can conclude that

$$
\lim _{D \cap \Gamma \ni \rightarrow 0} F_{D_{\xi}}((z, w), Y)=F_{D_{0}}((z, w), Y) \quad \forall(z, w) \in D_{0}, Y \in \mathbb{C}^{n+1} .
$$

Moreover the convergence takes place uniformly on compact sets of $D_{0} \times \mathbb{C}^{n+1}$. If we take $Y=X(\xi)$ in the above equation, we get

$$
\lim _{D \cap \Gamma \ni \xi \rightarrow 0} F_{D_{\xi}}((z, w), X(\xi))=F_{D_{0}}((z, w), X(0)) \quad \forall(z, w) \in D_{0} .
$$

Now, by the definitions of $D_{\xi}$ and $\Phi_{\xi}$, we have clearly

$$
\Phi_{\xi}\left(D_{\xi}\right)=D \cap U \text { and } \Phi_{\xi}(0)=\xi .
$$

It then follows from the invariance of the metric, (5.14) and (5.15), that

$$
\lim _{D \cap \Gamma \ni \xi \rightarrow 0} F_{D \cap U}\left(\xi, \Phi_{\xi *}(X(\xi))\right)=F_{D_{0}}(0, X(0)) .
$$


This is exactly what we need to prove if we notice that $\Phi_{\xi_{*}}=\left(\Pi_{|r(\xi)|}\right)_{*}$. This completes the proof of Theorem 5.1.

Finally, let us put all the pieces of information together to finish the proof of our main theorem stated in $\S 1$.

Proof of main theorem. Let $r(z)$ be a local defining function for $\Omega$ near $p$. Then by Definition 1.3 ([CA2]), there is a neighborhood $U$ of $p$ in $\mathbb{C}^{n+1}$ and a biholomorphic mapping $H_{p}(z)$ on $U$, such that in the new coordinates $p=0$ and $r(q)=: r\left(H_{p}^{-1}(q)\right)$ has the expansion

$$
r(q)=\operatorname{Re} q_{n+1}+P\left(q^{\prime}\right)+Q(q), \quad \text { for } q=\left(q^{\prime}, q_{n+1}\right) \in H_{p}^{-1}(U) .
$$

Here $P$ is a $\Lambda$-homogeneous polynomial and $Q$ is the remaining term in the expansion.

Let $D=H_{p}(\Omega \cap U)$. Then we may apply Lemmas 4.10 and 4.11 to find a biholomorphic mapping $\Psi$ (we may assume, by shrinking $U$ if necessary, that $\Psi$ is defined on $D$ ) of the form

$$
\begin{aligned}
\Psi: \quad \tilde{z}_{i} & =q_{i}, \quad 1 \leq i \leq n, \\
& \tilde{w}=q_{n+1}+b_{1}\left(q^{\prime}\right) q_{n+1}+b_{2}\left(q^{\prime}\right) q_{n+1}^{2}+b_{3}\left(q^{\prime}\right),
\end{aligned}
$$

where $b_{1}, b_{2}, b_{3}$ are smooth functions of $q^{\prime}$ satisfying $b_{i}=O\left(q^{\prime 2}\right), i=1,3$, such that $\Psi(D)=: \tilde{D}$ satisfies all hypotheses of Theorem 5.1. Put $\tilde{\Gamma}=\Psi\left(H_{p}(\Gamma)\right)$ and $\xi=\Psi(q)=\Psi\left(H_{p}(z)\right)$. Then it is easy to see that $\tilde{\Gamma}$ is a nontangential cone in $\tilde{D}$ with vertex at 0 and $\xi \rightarrow 0$ lying $\tilde{D} \cap \tilde{\Gamma}$ when $D \cap \Gamma \ni q \rightarrow 0$. Moreover, we have

$$
\lim _{D \cap\lceil\ni q \rightarrow 0} \frac{d(q)}{t}=1, \quad \text { where } t=|r(\xi)|=|r(\Psi(q))| .
$$

Now we perform the rescaling $\Phi_{\xi}$ as defined by (5.6) and set $D_{\xi}=\Phi_{\xi}^{-1}(\tilde{D})$ as before. Then it follows from the proof of Theorem 5.1 that $D_{\xi} \rightarrow D_{p}$ as $\xi \rightarrow 0$ in $\tilde{D} \cap \tilde{\Gamma}$. Further, by Theorem 4.7 , there is a taut domain $\Omega$ such that $D_{\xi} \subset \Omega$ for all $\xi \in \tilde{D} \cap \tilde{\Gamma}$. Theorem 2.1 then implies that

$$
\lim _{\tilde{D} \cap \tilde{\Gamma} \ni \xi \rightarrow 0} F_{D_{\xi}}(0, Y(\xi))=F_{D_{p}}(0, Y(0)),
$$

for any mapping $Y(\xi)$ from $\tilde{D} \cap \tilde{\Gamma}$ to $\mathbb{C}^{n+1}$ which is continuous at 0 . Let $X(z)$ be given as in the hypothesis, and set $\tilde{X}(z)=H_{p *}(X(z))$ and $\tilde{X}(q)=\Pi_{d_{*}} \tilde{X}(z)$ where $d=\operatorname{dist}(q, \partial D)$. Let $Y(\xi)=\left(Y_{1}(\xi), \ldots, Y_{n+1}(\xi)\right)=\Phi_{\xi *}^{-1} \Psi_{*}(\tilde{X}(q))$. An easy calculation using (5.17) and the definition of $\tilde{X}(q)$ gives, as $z \rightarrow p$ in $\Omega \cap \Gamma$, that

$$
\begin{aligned}
Y_{i}(\xi) & =t^{-1} d^{\lambda_{i}} \tilde{X}_{i}(z) \rightarrow \tilde{X}_{i}(p), \quad \text { by }(5.18), \text { for } i=1, \ldots, n \\
Y_{n+1}(\xi) & =\sum_{i=1}^{n} B_{i}(q) t^{-1} d^{\lambda_{i}} \tilde{X}_{i}(z)+B_{n+1}(q) t^{-1} d \tilde{X}_{n+1}(z) \rightarrow \tilde{X}_{n+1}(p)
\end{aligned}
$$

Here, in (5.21), the $B_{i}$ 's are smooth functions satisfying $B_{i}(q)=O\left(q^{2}\right)$ for $i=1, \ldots, n$ and $B_{n+1}(q) \rightarrow 1$ as $q \rightarrow 0$. Formula (5.21) holds because of 
(5.18) and the fact that $|q| \lesssim d(q)$. Hence we get $Y(\xi) \rightarrow \tilde{X}(p)$ as $q \rightarrow 0$ in $D \cap H_{p}(\Gamma)$.

On the other hand, since $\Phi_{\xi}^{-1} \Psi(q)=0$, by the invariance of the metric we obtain

$$
\begin{aligned}
F_{D}(q, \tilde{X}(q)) & =F_{\Phi_{\xi}^{-1} \Psi(D)}(0, Y(\xi)) \\
& =F_{D_{\xi}}(0, Y(\xi)) \rightarrow F_{D_{p}}(0, \tilde{X}(p)), \quad \text { as } q \rightarrow 0 \text { in } D \cap H_{p}(\Gamma) .
\end{aligned}
$$

By the definitions of $D$ and $H_{p}(z)$ we get

$$
\begin{aligned}
F_{D}(q, \tilde{X}(q)) & =F_{H_{p}(\Omega \cap U)}(q, \tilde{X}(q))=F_{\Omega \cap U}\left(z,\left(H_{p *}^{-1}\right) \tilde{X}(q)\right) \\
& =F_{\Omega \cap U}\left(z, H_{p *}^{-1} \Pi_{d *} H_{p *} X(z)\right) .
\end{aligned}
$$

Finally combining the localization result Theorem 2.2, (5.22) and (5.23) we obtain

$$
\lim _{\Omega \cap\lceil\ni z \rightarrow p} F_{\Omega}\left(z, H_{p *}^{-1} \Pi_{d *} H_{p *} X(z)\right)=F_{D_{p}}(0, \tilde{X}(p))=F_{D_{p}}\left(0,\left(H_{p}\right)_{* p}(X(p))\right) .
$$

This completes the proof of our Main Theorem.

Remark. (i) The nontangential condition in both the Main Theorem and Theorem 5.1 cannot be removed (cf. [GR]).

(ii) Even though we did not specify the optimal smoothness for the boundary throughout the proof of the Main Theorem, it is quite clear from our discussion that the least smoothness which is sufficient for our arguments to hold is that $\partial \Omega$ is $C^{\Delta_{1}(p)}$ smooth near the boundary point $p$.

Now we derive a few easy consequences of the Main Theorem and of Theorem 5.1.

Corollary 5.2. Let $\Omega, p, D_{p}, \Gamma$ be given as in the Main Theorem. Then, for any $X \in \mathbb{C}^{n+1}$,

(1) if $X_{N}(p) \neq 0$, we have

$$
\lim _{\Omega \cap \Gamma \ni \xi \rightarrow p} F_{\Omega}(z, X) d_{\Omega}(z)=F_{D_{p}}\left(0, X_{N}(p)\right) ;
$$

(2) if $X_{N}(p)=0$, that is $X \in T_{p}^{c}(\partial \Omega)$, we have

$$
\lim _{\Omega \cap \Gamma \ni z \rightarrow p} F_{\Omega}\left(z,\left(H_{p}\right)_{* z}\left(\Pi_{d_{\Omega}(z)}\right)_{* q}\left(H_{p}\right)_{* z} X\right)=F_{D_{p}}(0, X) .
$$

Here $X_{N}(p)$ is the complex normal component of $X$ at $p: X_{N}(p)=$ $\sum_{i=1}^{n+1} \frac{\partial r}{\partial z_{i}}(p) X_{i}, r$ is a local defining function for $\Omega$ near $p$ and $T_{p}^{c}(\partial \Omega)$ stands for the complex tangent space at $p$.

Proof. To prove (1), we may use the Main Theorem, setting

$$
V(z)=\left(H_{p}\right)_{* z}^{-1}\left(\Pi_{d_{\Omega}(z)}\right)_{* q}^{-1}\left(H_{p}\right)_{* z}\left(d_{\Omega}(z) X\right) .
$$

Observe that a biholomorphism maps a complex tangent vector to a complex tangent vector, it is easy to check that $V(z) \rightarrow\left(H_{p}\right)_{* p}^{-1} X_{N}(p)$ as $z \rightarrow p$. To prove (2), we simply put $V(z) \equiv X$ and apply the Main Theorem. 
Corollary 5.3. Let $\Omega$ be a taut domain in $\mathbb{C}^{n+1}$ and $p \in \partial \Omega$ a strongly pseudoconvex point. Let $r$ be a local $C^{2}$ defining function. Then for any nontangential cone $\Gamma \subset \Omega$ with vertex at $p$, we have

(1) if $X_{N}(p) \neq 0$, then

$$
\lim _{\Omega \cap \Gamma \ni z \rightarrow p} F_{\Omega}(z, X)|r(z)|=\left|X_{N}(p)\right| ;
$$

(2) if $X_{N}(p)=0$, i.e., $X \in T_{p}^{c}(\partial \Omega)$, then

$$
\lim _{\Omega \cap \Gamma \ni z \rightarrow p}\left(F_{\Omega}(z, X)\right)^{2}|r(z)|=L_{r}(p, X) .
$$

Here $L_{r}(p, X)$ is the Levi form of $r$ at $p$. This result was first obtained by Graham [GR]. Note here that the metric $F_{\Omega}$ can be any of the Kobayashi metrics as well as the Carathéodory metric, simply because near a strongly pseudoconvex point the domain is convexible and thus all these metrics are the same (cf. [LE], [R-W]).

Corollary 5.4. Let $\Omega$ be a taut domain in $\mathbb{C}^{n+1}$ and $0 \in \partial \Omega$ with $\mathscr{M}(0)=$ $\left(1, m_{1}, \ldots, m_{n}\right), m_{n}<\infty$. Assume that $\Omega$ has a defining function $r(z, w)$ near 0 of the form $r(z, w)=\operatorname{Re} w+P(z)+Q(z, w)$, where $P$ is $\Lambda$-homogeneous p.s.h. circular polynomial such that $P$ is not harmonic on any complex line through 0 , and $Q \in \mathscr{O}(1+\varepsilon,(\Lambda, 1))$, for some $\varepsilon>0$. Then for any nontangential cone $\Gamma \subset \Omega$ with vertex at 0 , we have

$$
\begin{aligned}
& \lim _{\Omega \cap \Gamma \ni(z, w) \rightarrow 0} F_{\Omega}((z, w), X)|r(z, w)|=\left|X_{N}\right| \\
& \lim _{\Omega \cap \Gamma \ni(z, w) \rightarrow 0} F_{\Omega}\left((z, w), \pi_{r *}(X)\right)=\left(\lambda_{P}\left(X_{T}\right)\right)^{-1}, \quad \forall X \in T_{0}(\partial \Omega) .
\end{aligned}
$$

Here, $F_{\Omega}$ is any of the Kobayashi metrics, $X_{N}=\left(0, X_{n+1}\right), X_{T}=\left(X_{1}, \ldots\right.$, $\left.X_{n}\right)$ and $\lambda_{P}\left(X_{T}\right)$ is the unique positive solution to the equation $P\left(\lambda X_{T}\right)=1$. By " $P$ is circular" we mean

$$
P(\zeta z)=P(z), \quad \forall z \in \mathbb{C}^{n}, \zeta \in \partial \Delta .
$$

Proof. First of all, we prove that the equation $P\left(\lambda X_{T}\right)=1$ has a unique positive solution by showing that the polynomial $P(z)$ has the following properties:

(1) $P(z) \geq 0$ for all $z \in \mathbb{C}^{n}$ and $P(z)=0$ if and only if $z=0$;

(2) the function $\mathbb{R}^{+} \ni \lambda \rightarrow P(\lambda z)$ is strictly increasing for any fixed $z \neq 0$. Since $P$ is circular in the sense of (5.25) we have, for any $0 \neq z \in \mathbb{C}^{n}$ fixed,

$$
P(z)=\frac{1}{2 \pi} \int_{0}^{2 \pi} P(z) d \theta=\frac{1}{2 \pi} \int_{0}^{2 \pi} P\left(e^{i \theta} z\right) d \theta \geq 0 .
$$

The last inequality follows from the submean value property. So $P(z) \geq 0$ for all $z \in \mathbb{C}^{n}$. Moreover, since each $P\left(e^{i \theta} z\right) \geq 0, P(z)=0(z \neq 0)$ would imply by (5.26) that $P(\zeta z)=0$ on $\{\zeta:|\zeta|=1\}$; then it would follow from the maximum principle that $P(\zeta z) \equiv 0$, which contradicts our hypothesis on $P$. This proves (1).

To see (2), let $\lambda_{1}<\lambda_{2}$ and $z \neq 0$. Since $P(\zeta z)$ is subharmonic on $\{\zeta$ : $\left.|\zeta|<\lambda_{2}\right\}$, the maximum principle together with (5.25) implies that

$$
P\left(\lambda_{1} z\right)=\max _{|\zeta|=\lambda_{1}} P(\zeta z) \leq \max _{|\zeta|=\lambda_{2}} P(\zeta z)=P\left(\lambda_{2} z\right) \text {. }
$$


If $P\left(\lambda_{1} z\right)=P\left(\lambda_{2} z\right)$, then the same principle shows that $P(\zeta z) \equiv P(0)=0$, which again contradicts our hypothesis. Note also that, for $X_{T} \neq 0, P\left(\lambda X_{T}\right)$ is a polynomial in $\lambda$. Thus, from (1) and (2), we must have that $\lim _{\lambda \rightarrow \infty} P\left(\lambda X_{T}\right)=$ $\infty$. This is enough to see that the equation $P\left(\lambda X_{T}\right)=1$ has a unique positive solution.

Now because of $(1)$, the model $D_{p}=\{(z, w): \operatorname{Re} w+P(z)<1\}$ is positive and thus h-extendible (cf. Example 3.4). Hence $(\Omega, p)$ is h-extendible. By Corollary 5.2, in order to prove our corollary, it suffices to show that

$$
F_{D_{p}}\left(0, X_{N}\right)=\left|X_{N}\right| \text { and } F_{D_{p}}\left(0, X_{T}\right)=1 / \lambda_{P}\left(X_{T}\right) .
$$

Here we are identifying $X_{T}$ with $\left(X_{1}, \ldots, X_{n}, 0\right)$. To this end, let $F$ be a $k$-th order Kobayashi metric for $\Omega$. Observe that the complex discs $\Delta \ni \zeta \rightarrow \zeta^{k} \frac{X_{N}}{\left|X_{N}\right|}$ and $\Delta \ni \zeta^{k} \rightarrow L_{P}(X) \zeta X_{T}$ lie in $D_{p}$ (to check that the second one lies in the model, one has to use (2)). In light of the definition of the metric, we get

$$
F_{D_{p}}\left(0, X_{N}\right) \leq\left|X_{N}\right| \text { and } F_{D_{p}}\left(0, X_{T}\right) \leq 1 / \lambda_{P}\left(X_{T}\right) .
$$

On the other hand, by $(1), D_{p} \subset\{(z, w): \operatorname{Re} w<1\}$. Hence it is easy to obtain $F_{D_{p}}\left(0, X_{N}\right) \geq\left|X_{N}\right|$. This yields the first equation of (5.27). For the second one, we let $\tilde{\varphi}=\left(\varphi, \varphi_{n+1}\right)$ be any complex disc in $D_{p}$ with

$$
\nu(\tilde{\varphi})=k, \quad \text { and } \quad(\tilde{\varphi})^{(k)}(0)=k ! \lambda X_{T} .
$$

We may write $\varphi(\zeta)=\zeta^{k} \psi(\zeta)$ where $\psi$ is holomorphic on $\Delta$. Clearly, $\psi(0)=$ $\lambda X_{T}$. Since $\tilde{\varphi}(\Delta) \subset D_{p}$, we have $\operatorname{Re} \varphi_{n+1}(\zeta)+P\left(\zeta^{k} \psi(\zeta)\right)<1$ for all $\zeta \in \Delta$. It follows that, for any $0<\delta<1$,

$$
\frac{1}{2 \pi} \int_{|\zeta|=r} \operatorname{Re} \varphi_{n+1}(\zeta) d \zeta+\frac{1}{2 \pi} \int_{|\zeta|=r} P\left(\zeta^{k} \psi(\zeta)\right) d \zeta \leq 1 .
$$

The first integral is zero because of harmonicity. By (5.25) the second one can be written as

$$
\begin{aligned}
\frac{1}{2 \pi} \int_{|\zeta|=r} P\left(|\zeta|^{k} \psi(\zeta)\right) d \zeta & =\frac{1}{2 \pi} \int_{|\zeta|=r} P\left(\delta^{k} \psi(\zeta)\right) d \zeta \\
& \geq P\left(\delta^{k} \psi(0)\right)=P\left(\delta^{k} \lambda X_{T}\right) .
\end{aligned}
$$

The inequality in (5.31) follows from the submean value property. Combining (5.30) and (5.31), we obtain $P\left(\delta^{k} \lambda X_{T}\right) \leq 1$ for all $0<\delta<1$. Letting $\delta \rightarrow$ $1^{-}$, we have $P\left(\lambda X_{T}\right) \leq 1=P\left(\lambda_{P}\left(X_{T}\right) X_{T}\right)$. By the strict monotonicity (2), $\lambda \leq \lambda_{P}\left(X_{T}\right)$. This implies that $F_{D_{p}}\left(0, X_{T}\right) \geq 1 / \lambda_{P}\left(X_{T}\right)$, as desired.

A few simple observations are in order: the first equality in Corollary 5.4 still holds if $P$ is only positive instead of circular; if $P$ is a homogeneous circular polynomial of degree $2 k$, then it is easy to get $\lambda_{P}\left(X_{T}\right)=\left(P\left(X_{T}\right)\right)^{-1 / 2 k}$. This gives in particular an alternative proof of Corollary 5.3. However, it is usually impossible to express explicitly the quantity $\lambda_{P}$ in terms of the coefficients of $P$. For instance, in case the polynomial is $P(z)=\sigma(z)=\sum_{1}^{n}\left|z_{i}\right|^{m_{i}}$, it follows that $\lambda_{P}\left(X_{T}\right)$ is the solution to the equation $\sum_{1}^{n} \lambda^{m_{i}}\left|X_{i}\right|^{m_{i}}=1$. Therefore the results such as Corollaries 5.2 and 5.4 are probably the most explicit relations between the weighted boundary limits of the Kobayashi metrics and the Levi polynomials that we could ever hope to obtain. On the other hand, it can 
be proved by using the stability property of the Kobayashi metrics that the weighted limits in Corollary 5.2 depend continuously on the Levi polynomial of the domain (cf. [YU5]).

\section{REFERENCES}

[BKM] B. Blank, D. Klein, S. Krantz, D. Fan, D. Ma, and M. Pang, The Kobayashi metric of complex ellipsoids, Experimental Math. 1 (1992), 47-55.

[BP1] E. Bedford and S. Pincuk, Domains with noncompact automorphism groups, J. Geometric Anal. 1 (1991), 165-191.

[BP2] _ Convex domains with noncompact automorphism groups, Math. Sb.-USSR (to appear).

[CA1] D. Catlin, Estimates of invariant metrics on pseudoconvex domains of dimension two, Math. Z. 200 (1989), 429-466.

[CA2] - Boundary invariants of pseudoconvex domains, Ann. of Math. 120 (1984), 529-586.

[CHE] J. Chen, Estimates of the invariant metrics on convex domains, Purdue University Ph.D. Dissertation, 1989.

[CHO] S. Cho, A lower bound on the Kobayashi metric near a point of finite type, J. Geometric Anal. 2 (1992), 317-326.

[DA1] J. D'Angelo, Real hypersurfaces, order of contact, and applications, Ann. of Math. 115 (1982), 615-637.

[DA2] - Several complex variables and geometry, CRC Press, Boca Raton, 1992.

[D-F] K. Diederich and J. Fornæss, Proper holomorphic maps onto pseudoconvex domains with real analytic boundary, Ann. of Math. 110 (1979), 575-592.

[DE] J. P. Demailly, Un exemple fibré holomorphe non de Stein á fibre $\mathbb{C}^{2}$ ayant pour base le disque ou le plan, Invent. Math 48 (1978), 293-302.

[DHO] K. Diederich, G. Herbort and T. Ohsawa, The Bergman kernel on uniformly extendible pseudoconvex domains, Math. Ann. 273 (1986), 471-478.

[D-L] K. Diederich and I. Lieb, Konvexitaet in der komplexen analysis, Birkhäuser, Boston, 1981.

[FE] C. Fefferman, The Bergman kernel and biholomorphic mappings of pseudoconvex domains, Invent. Math. 26 (1974), 1-65.

[F-R] F. Forstneric and J. Rosay, Localizations of the Kobayashi and the boundary continuity of proper holomorphic mappings, Math. Ann. 279 (1987), 239-252.

[FR] S. Frankel, Applications of affine geometry to geometric function theory in several complex variables, Proc. Sympos. Pure Math., vol. 52, Amer. Math. Soc., Providence, RI, 1991, pp. 183-208.

[F-S] J. Fornæss and N. Sibony, Construction of p.s.h. functions on weakly pseudoconvex domains, Duke Math. J. 58 (1989), 633-655.

[GK1] R. Greene and S. Krantz, Stability of the Caratheodory and Kobayashi and applications to biholomorphic mappings, Proc. Sympos. Pure Math., vol. 41, Amer. Math. Soc., Providence, RI, 1984, pp. 77-93.

[GK2] Deformation of complex structures, estimates for the $\bar{\partial}$-equation and stability of the Bergman kernel, Adv. Math. 43 (1982), 1-86.

[GR] I. Graham, Boundary behavior of the Carathéodory and the Kobayashi on strongly pseudoconvex domains with smooth boundary, Trans. Amer. Math. Soc. 207 (1975), 219-240.

[HE] G. Herbort, Invariant metrics and peak functions on pseudoconvex domain of homogeneous finite diagonal type, Math. Z. 209 (1992), 223-243.

[HO] L. Hormander, Introduction to complex analysis in several variables, 3rd ed., 1990.

[H-S] M. Hakim and N. Sibony, Quelques conditions pour l'existence de fonctions pics dans des domains pseudoconvexes, Duke Math. J. 44 (1977), 399-406.

[J-P] M. Jarnicki and P. Pflug, Invariant distances and metrics in complex analysis, De Gruyter, New York, 1993. 
[KA] M. Kalka, Deformation of the Kobayashi metric, Proc. Amer. Math. Soc. 59 (1976), 245251.

[KI] K. Kim, Complete localization of domains with noncompact automorphism groups, Trans. Amer. Math. Soc. 319 (1990), 130-153.

[KO1] S. Kobayashi, Intrinsic distances, measures and geometric function theory, Bull. Amer. Math. Soc. (1976), 357-416.

[KO2] - Hyperbolic manifolds and holomorphic mappings, Dekker, New York, 1970.

[KON] J. Kohn, Boundary behavior of $\bar{\partial}$ on weakly pseudoconvex manifolds of dimension two, $\mathrm{J}$. Differential Geometry 6 (1972), 523-542.

[K-R] N. Kerzman and J. Rosay, Fonctions plurisubharmonique d'exhaustion bornées et domaines taut, Math. Ann. 257 (1982), 171-184.

[KR1] S. Krantz, Function theory of several complex variables, 2nd ed., Wadsworth, Belmont, 1992.

[KR2] - The boundary behavior of the Kobayashi metric, Rocky Mountain J. Math. 22 (1992), 227-233.

[KR3] - Geometric analysis and function spaces, CBMS Lecture Notes, A.M.S., 1993.

[KR4] _ Invariant metrics and the boundary behavior of holomorphic functions on domains in $\mathbb{C}^{n}$, J. Geometric Anal. 1 (1991), 71-97.

[K-Y] K. Kim and J. Yu, Boundary behavior of the Bergman curvature in the strongly pseudoconvex polyhedral domains, preprint.

[LE] L. Lempert, La metrique Kobayashi et las representation des domains sur la boule, Bull. Soc. Math. France 109 (1981), 427-474.

[MC] J. McNeal, Local geometry of decoupled pseudoconvex domains, Proceedings in honor of Hans Grauert, Aspekte der Mathematik, Berlin, 1990, pp. 223-230.

[NO] A. Noell, Peak functions for pseudoconvex domains, Several Complex Variables: Proceedings of the Mittag-Leffler Institute, 1987-1988, Annals of Mathematics Studies, Princeton, NJ, 1993.

[PI] S. Pincuk, The scaling method and holomorphic mappings, Proc. Sympos. Pure Math., vol. 52, Amer. Math. Soc., Providence, RI, 1991, pp. 151-163.

[RAM] I. Ramadanov, Some applications of the Bergman kernel to geometric theory of functions, Complex Analysis, Banach Center Publications, vol. 11, Warsaw 1983.

[R-W] H. Royden and P. Wong, Carathéodory and Kobayashi metric on convex domains, preprint, 1983.

[ROY] H. Royden, Remarks on the Kobayashi metric, Lecture Notes in Math., vol. 185, Springer, Berlin, 1971, pp. 125-137.

[SI1] N. Sibony, Some aspects of weakly pseudoconvex domains, Proc. Sympos. Pure Math., vol. 52, Amer. Math. Soc., Providence, RI, 1991, pp. 199-233.

[SI2] _ A class of hyperbolic manifolds, Ann. of Math. Studies 10 (1981), 257-272.

[YU1] J. Yu, Multitypes of convex domains, Indiana Univ. Math. J. 41 (1992), 837-849.

[YU2] - Invariant metrics and finite type conditions, Proc. Amer. Math. Soc. (to appear).

[YU3] - Stability of the Bergman kernel and metric, preprint.

[YU4] _., Peak functions on weakly pseudoconvex domains, preprint.

[YU5] _ Geometric analysis on weakly pseudoconvex domains, Dissertation, Washington University, 1993.

Department of Mathematics, Texas A\&M University, College Station, Texas 77843 E-mail address: jyu@math.tamu.edu 